\title{
Uniformly factoring weakly compact operators and parametrised dualisation
}

\author{
L. Antunes ${ }^{1}$, K. Beanland ${ }^{2}$ and B. M. Braga ${ }^{3}$ \\ ${ }^{1}$ Departamento de Matemática, Universidade Tecnológica Federal do Paraná, Campus Toledo, 85902-490 Toledo, PR Brazil; \\ E-mail: leandroantunes@utfpr.edu.br. \\ ${ }^{2}$ Department of Mathematics, Washington \& Lee University, 204 W. Washington St. Lexington, VA, 24450; \\ E-mail: beanlandk@wlu.edu. \\ ${ }^{3}$ Department of Mathematics, University of Virginia, 141 Cabell Drive, Kerchof Hall P.O. Box 400137 Charlottesville, VA \\ 22904; E-mail: demendoncabraga@gmail.com.
}

Received: 10 July 2020; Revised: 1 December 2020; Accepted: 14 December 2020

2020 Mathematics Subject Classification: Primary - 46B10; Secondary - 03E15

\begin{abstract}
This article deals with the problem of when, given a collection $\mathcal{C}$ of weakly compact operators between separable Banach spaces, there exists a separable reflexive Banach space $Z$ with a Schauder basis so that every element in $\mathcal{C}$ factors through $Z$ (or through a subspace of $Z$ ). In particular, we show that there exists a reflexive space $Z$ with a Schauder basis so that for each separable Banach space $X$, each weakly compact operator from $X$ to $L_{1}[0,1]$ factors through $Z$.

We also prove the following descriptive set theoretical result: Let $\mathcal{L}$ be the standard Borel space of bounded operators between separable Banach spaces. We show that if $\mathcal{B}$ is a Borel subset of weakly compact operators between Banach spaces with separable duals, then for $A \in \mathcal{B}$, the assignment $A \rightarrow A^{*}$ can be realised by a Borel $\operatorname{map} \mathcal{B} \rightarrow \mathcal{L}$.
\end{abstract}

\section{Contents}

1 Introduction

2 Preliminaries

2.1 Polish Spaces and Coding Separable Banach Spaces . . . . . . . . . . . . . . . . 3

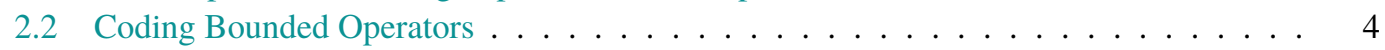

2.3 Isometry and Isomorphism of Operators . . . . . . . . . . . . . . . . 5

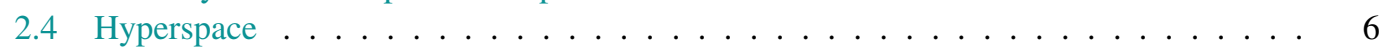

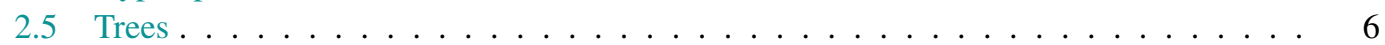

3 The Adjoint Map as a Borel Function $\quad 6$

3.1 Consequences of Theorem $3.1 \ldots \ldots \ldots \ldots \ldots \ldots$

4 Factoring Weakly Compact Operators Through a Single Space 12

5 Factoring Weakly Compact Operators Through Subspaces of a Single Space 18

5.1 Factoring a Borel $\mathcal{B} \subset \mathcal{W}_{\mathrm{SB}, \mathrm{SD}}$ Through a Family of Reflexive Spaces with Bases . . . 19

5.2 Coding by Rational Spaces and Amalgamation . . . . . . . . . . . . . . 21

6 Concluding Remarks and Questions $\quad 25$

(c) The Author(s), 2021. Published by Cambridge University Press. This is an Open Access article, distributed under the terms of the Creative Commons Attribution licence (http://creativecommons.org/licenses/by/4.0/), which permits unrestricted re-use, distribution, and reproduction in any medium, provided the original work is properly cited. 


\section{Introduction}

The celebrated W. Davis, T. Figiel, W.B. Johnson and A. Pełczyński factorisation theorem states that every weakly compact bounded operator factors through a reflexive space [15, Corollary 1]: Recall that a bounded operator $A: X \rightarrow Y$ between Banach spaces $X$ and $Y$ is called weakly compact if the closure of the image of the unit ball of $X$ under $A$ is compact with respect to the weak topology of $Y$; furthermore, the operator $A$ is said to factor through a Banach space $Z$ if there are bounded operators $B: X \rightarrow Z$ and $C: Z \rightarrow Y$ so that $A=C \circ B$. This result has been used and generalised in many different directions in the past 45 years. A natural generalisation, considered by several authors, asks when a uniform factorisation is possible in the following sense: For which collections $\mathcal{C}$ of weakly compact operators between separable Banach spaces does there exist a separable reflexive space $Z$ so that every operator in $\mathcal{C}$ factors through $Z$ ? As we explain later, a uniform factorisation of this type is not possible for all weakly compact operators between separable Banach spaces.

Our main uniform factorisation theorem is the following.

Theorem A. There is a separable reflexive space with a Schauder basis $Z$ so that if $X$ is a separable Banach space, $Y$ isomorphic to $L_{1}[0,1]$ and $A: X \rightarrow Y$ is a weakly compact operator, then A factors through $Z$.

The proof of the above theorem uses methods from descriptive set theory and complexity results. In particular, we do not explicitly define the space $Z$ and, in fact, have very little concrete information about the structure of this space. In addition to Theorem A, we obtain many other uniform factorisation-type theorems for certain collections of weakly compact operators between separable $\mathrm{Ba}$ nach spaces; however, to state these results, we will require some terminology from descriptive set theory.

First, allow us to further motivate this inquiry by explaining some known obstructions to uniform factorisations for weakly compact operators. In [28, Theorem 3.3], W. Szlenk proved that no separable reflexive space contains isomorphic copies of all separable reflexive Banach spaces. Consequently, there is no separable reflexive space through which every weakly compact operator between separable Banach spaces factors. Therefore, uniform factorisation questions are only relevant for proper subclasses of weakly compact operators. As is often common for this kind of result, descriptive settheoretic tools provide us the appropriate framework to study which subclasses have this property (cf. $[7,9,18]$ ).

There is another natural obstruction for factorisation theorems for weakly compact operators. Because a complemented subspace of a Banach space with a Schauder basis must have the bounded approximation property, if $\mathcal{C}$ is a collection of operators that contains the identity on a reflexive space without the bounded approximation property and $Z$ is a space so that every operator in $\mathcal{C}$ factors through, then $Z$ cannot have a basis. It is therefore natural to restrict our attention to weakly compact operators that factor through spaces with bases. This, in turn, focusses our attention on weakly compact operators defined on spaces whose domains or codomains have a basis.

In [9] the second named author and D. Freeman initiated the program of studying uniform factorisation problems using descriptive set theory. For that, the authors introduced a natural coding for the class of all operators between arbitrary Banach spaces, denoted by $\mathcal{L}$; that is, $\mathcal{L}$ is a standard Borel space that naturally codes this class of operators (we refer the reader to Section 2 for precise definitions). This coding was also used in the papers $[7,4,5,6,12]$. Because the coding space $\mathcal{L}$ is a standard Borel space, this allows one to study the complexity of classes of bounded operators between separable Banach spaces; that is, we can inquire whether certain classes of operators are Borel, analytic, coanalytic, etc.

The main result of [9] (Theorem 25) states that if $\mathcal{A}$ is an analytic subset of weakly compact operators between separable Banach spaces so that either

1. for all $T: X \rightarrow Y$ in $\mathcal{A}, Y$ has a shrinking basis or

2. for all $T: X \rightarrow Y$ in $\mathcal{A}, Y$ is isomorphic to $C(\Delta)$, where $\Delta$ is the Cantor set, 
then there exists a separable reflexive Banach space $Z$ admitting Schauder basis such that every operator in $\mathcal{A}$ factors through $Z$. In Section 4 (Theorem 4.3), we extend this result to several other classes of weakly compact operators. Precisely, we obtain the following.

Theorem B. Let $\mathcal{A}$ be an analytic subset of weakly compact operators between separable Banach spaces so that either

1. for all $T: X \rightarrow Y$ in $\mathcal{A}, Y$ has an unconditional basis;

2. for all $T: X \rightarrow Y$ in $\mathcal{A}, X^{*}$ has an unconditional basis and $Y$ has separable dual or

3. for all $T: X \rightarrow Y$ in $\mathcal{A}, X^{*}$ has a shrinking basis and $Y$ has separable dual.

Then there exists a separable reflexive Banach space $Z$ with a Schauder basis so that every operator in $\mathcal{A}$ factors through $Z$.

Our main tool to prove Theorem B for $\mathcal{A}$ satisfying (2) or (3) therein is a new descriptive set-theoretic result (Theorem 3.1) that extends a theorem by the third named author (see [11, Theorem 1.1]). Precisely, let $\mathcal{L}_{\mathrm{SD}}$ denote the subset of $\mathcal{L}$ that codes all ofthe bounded operators $A: X \rightarrow Y$ between Banach spaces $X$ and $Y$ with separable dual; we prove in Theorem 3.1 that if $\mathcal{B}$ is a Borel subset of $\mathcal{L}_{\mathrm{SD}}$ then there exists a Borel assignment $A \in \mathcal{B} \mapsto A^{\bullet} \in \mathcal{L}$ such that $A^{\bullet}$ is isometric to $A^{*}$ (see Definition 2.3).

In addition to being used to prove Theorem 4.3, Theorem 3.1 has other consequences that are interesting on their on. For instance, in Corollary 3.6 we prove that if $\mathcal{A} \subset \mathcal{L}_{\mathrm{SD}}$ is analytic, then the set

$$
\mathcal{A}^{*}=\left\{B \in \mathcal{L}: \exists A \in \mathcal{A} \text { with } B \text { is isomorphic to } A^{*}\right\}
$$

is also analytic. It is a generalisation of the main result of [17].

As noticed by G. Godefroy in [20, Problem 5.2], the result [11, Theorem 1.1] implies that the equivalence class $\langle X\rangle$ of a separable reflexive Banach space is Borel if and only if the equivalence class of its dual $\left\langle X^{*}\right\rangle$ is Borel. Another consequence of Theorem 3.1 is an analogous result for bounded operators, as we will see in Corollary 3.7.

Finally, in Section 5 we deal with strongly boundedness over the class of reflexive spaces. The second named author and R. Causey proved in [7, Theorem 5.8] that the class of weakly compact operators is strongly bounded over the class of separable reflexive Banach spaces. In Theorem 5.1 we prove that for an analytic subset $\mathcal{A}$ of the class of weakly compact operators $T$, with domain separable and codomain with separable dual, one can find a reflexive $Z$ so that each $A \in \mathcal{A}$ factors through a subspace of $Z$; moreover, the choice of this subspace can be done in a $\sigma\left(\Sigma_{1}^{1}\right)$-measurable manner.

\section{Preliminaries}

The Banach space theory terminology used herein is standard and we refer the reader to [2]. We emphasise here that all Banach spaces are considered to be over the reals and, given a Banach space $X$, we denote its closed unit ball by $B_{X}$. We also write $X \cong Y$ and $X \equiv Y$ to denote that $X$ and $Y$ are isomorphic and isometric, respectively. For the background on descriptive set theory, we refer the reader to [24].

\subsection{Polish Spaces and Coding Separable Banach Spaces}

A separable topological space $(X, \tau)$, where $\tau$ is a topology on the set $X$, is called a Polish space if there exists a complete metric on $X$ that generates the topology $\tau$. In this case, we say that $\tau$ is a Polish topology.

A measurable space $(X, \mathcal{A})$, where $\mathcal{A}$ is a $\sigma$-algebra on the set $X$, is called a standard Borel space if there exists a Polish topology on $X$ so that $\mathcal{A}$ is the Borel $\sigma$-algebra generated by this topology.

Because the class of all separable Banach spaces is a proper class - that is, it is not a set - in order to study the descriptive set-theoretic properties of the class of all separable Banach spaces it is 
necessary to first code this class as a set. This is usually done as follows. Because the Banach space of continuous real-valued functions on the Cantor set, $C(\Delta)$, is isometrically universal for all separable Banach spaces, ${ }^{1}$ we define

$$
\mathrm{SB}=\{X \subset C(\Delta): X \text { is a closed linear subspace }\}
$$

and endow SB with the Effros-Borel $\sigma$-algebra; that is, the $\sigma$-algebra generated by the sets

$$
\{\{F \in \mathrm{SB}: U \cap F \neq \emptyset\}: U \subset C(\Delta) \text { is open }\} .
$$

It is well known that SB endowed with the Effros-Borel structure is a standard Borel space [16, Theorem 2.2]. Similarly, if $Z \in \mathrm{SB}$, we define

$$
\mathrm{SB}(Z)=\{X \in \mathrm{SB}: X \subset Z\} .
$$

So $\mathrm{SB}(Z)$ is a Borel subset of SB and hence a standard Borel space.

With this coding in hand, it makes sense to ask whether specific classes of separable Banach spaces are Borel, analytic, coanalytic, etc. Moreover, any other 'reasonable coding' for the separable Banach spaces agrees with this coding (see [10, Proposition 2.8]).

\subsection{Coding Bounded Operators}

Let us introduce the coding for operators between separable Banach spaces defined in [9, Section 4] (cf. [4, Section 8.2]). Throughout this article, let

$$
\left(d_{n}: \mathrm{SB} \rightarrow C(\Delta)\right)_{n}
$$

be a sequence of Borel maps so that

(1) $\left\{d_{n}(X)\right\}_{n}$ is a dense subset of $X$ for all $X \in \mathrm{SB}$ and

(2) for all $m, k \in \mathbb{N}$ and all $r, q \in \mathbb{Q}$ there exists $n \in \mathbb{N}$ such that $d_{n}(X)=r d_{k}(X)+q d_{m}(X)$

(the existence of such a sequence is given by Kuratowski and Ryll-Nardzewski's selection theorem [24, Theorem 12.13]).

We use the notation $\mathbb{Q}_{>0}=\mathbb{Q} \cap(0, \infty)$ throughout.

Definition 2.1. We define the coding for all bounded operators between separable Banach spaces as the subset $\mathcal{L} \subset \mathrm{SB} \times \mathrm{SB} \times C(\Delta)^{\mathbb{N}}$ given by

$$
\begin{aligned}
& (X, Y, \hat{A}) \in \mathcal{L} \\
& \quad \Leftrightarrow(\forall n \in \mathbb{N}, \hat{A}(n) \in Y) \wedge\left(\exists L \in \mathbb{Q}_{>0}, \forall\left(a_{i}\right)_{i} \in \mathbb{Q}^{<\mathbb{N}},\left\|\sum_{i} a_{i} \hat{A}(i)\right\| \leqslant L\left\|\sum_{i} a_{i} d_{i}(X)\right\|\right) .
\end{aligned}
$$

Firstly, notice that $\mathcal{L}$ is clearly a Borel subset of $\mathrm{SB} \times \mathrm{SB} \times C(\Delta)^{\mathbb{N}}$ (see [4, Section 8.2] for details), so $\mathcal{L}$ is a standard Borel space. In addition, the definition above implies that if $d_{n}(X)=r d_{k}(X)+q d_{m}(X)$ for some $n, m, k \in \mathbb{N}$ and $r, q \in \mathbb{Q}$, then $\hat{A}(n)=r \hat{A}(k)+q \hat{A}(m)$.

We now explain how $\mathcal{L}$ codes the operators between separable Banach spaces. Given Banach spaces $X, Y \in \mathrm{SB}$ and a bounded operator $A: X \rightarrow Y$, we associate to $A: X \rightarrow Y$ the triple $(X, Y, \hat{A})$, where $\hat{A}=\left(A\left(d_{n}(X)\right)\right)_{n}$. Clearly, $(X, Y, \hat{A}) \in \mathcal{L}$. On the other hand, if $(X, Y, \hat{A}) \in \mathcal{L}$, define $A: X \rightarrow Y$ to be the unique operator such that $A\left(d_{n}(X)\right)=\hat{A}(n)$ for all $n \in \mathbb{N}$. For the remainder of this article, using the identification just described, we make no distinction between the triple $(X, Y, \hat{A}) \in \mathcal{L}$ and the

${ }^{1}$ That is, every separable Banach space embeds into $C(\Delta)$ isometrically [24, Page 79]. 
operator $A: X \rightarrow Y$ (cf. [4, Claim 8.4]). Moreover, if the spaces $X$ and $Y$ can be neglected, we simply write $A \in \mathcal{L}$.

Remark 2.2. We refer the interested reader to the two recent preprints [13, 14] in which the authors consider the space of norms on $c_{00}$ to provide a coding for separable Banach spaces. This newly developed framework can likely also be used to obtain a coding for $\mathcal{L}$.

Let $\mathrm{SD}=\left\{X \in \mathrm{SB}: X^{*}\right.$ is separable $\}$ and define

$$
\mathcal{L}_{\mathrm{SD}}=\{(X, Y, \hat{A}) \in \mathcal{L}: X, Y \in \mathrm{SD}\}
$$

Therefore, $\mathcal{L}_{\mathrm{SD}}=\mathcal{L} \cap\left(\mathrm{SD} \times \mathrm{SD} \times C(\Delta)^{\mathbb{N}}\right)$. Because SD is coanalytic [10, Corollary 3.3(ii)], so is $\mathcal{L}_{\mathrm{SD}}$. Moreover, let $\operatorname{Tr}$ be the standard Borel space of trees on $\mathbb{N}$ (see Subsection 2.5 for definitions regarding trees). Because SD is complete coanalytic (see the proof of [10, Corollary 3.3(ii)]), there exists a Borel reduction $\varphi: \operatorname{Tr} \rightarrow \mathrm{SB}$ of the well-founded trees, WF, to $\mathrm{SD}$; that is, $\varphi$ is a Borel map so that $\varphi^{-1}(\mathrm{SD})=$ WF. Define $\Phi: \operatorname{Tr} \rightarrow \mathcal{L}$ by letting

$$
\Phi(T)=\left(\varphi(T), \varphi(T),\left(d_{n}(\varphi(T))\right)_{n}\right)
$$

for all $T \in \operatorname{Tr}$. Then $\Phi$ is a Borel reduction of WF to $\mathcal{L}_{\mathrm{SD}}$. Therefore, $\mathcal{L}_{\mathrm{SD}}$ is also complete coanalytic. In particular, $\mathcal{L}_{\mathrm{SD}}$ is not Borel.

We denote the coding for the weakly compact operators by $\mathcal{W}$; that is,

$$
\mathcal{W}=\{A \in \mathcal{L}: A \text { is weakly compact }\}
$$

By [9, Proposition 22], $\mathcal{W}$ is coanalytic. Finally, we denote the coding for the weakly compact operators between spaces with separable dual by $\mathcal{W}_{\mathrm{SD}}$; that is,

$$
\mathcal{W}_{\mathrm{SD}}=\mathcal{W} \cap \mathcal{L}_{\mathrm{SD}}
$$

\subsection{Isometry and Isomorphism of Operators}

In [7, Definition 5.4], the authors defined the concept of two operators $A: X \rightarrow Y$ and $B: Z \rightarrow W$ being isomorphic to each other. We define analogously what it means for $A$ and $B$ to be isometric. We notice that, in order to obtain Theorem 4.3, we could simply work with the concept of isomorphic operators; however, because it is possible to obtain an isometric result in Theorem 3.1, we chose to work with isometric operators. We make both definitions below.

Definition 2.3. Let $X, Y, Z$ and $W$ be Banach spaces and $A: X \rightarrow Y$ and $B: Z \rightarrow W$ be bounded linear operators. We say that $A: X \rightarrow Y$ and $B: Z \rightarrow W$ are isometric (respectively isomorphic) if there exist linear isometries (respectively linear isomorphisms) $j_{1}: X \rightarrow Z$ and $j_{2}: Y \rightarrow W$ such that $B \circ j_{1}=j_{2} \circ A$; that is, such that the diagram

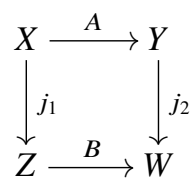

commutes. The pair $\left(j_{1}, j_{2}\right)$ is called an isometry (respectively isomorphism) between $A$ and $B$. We write $(A: X \rightarrow Y) \equiv(B: Z \rightarrow W)$ (respectively $(A: X \rightarrow Y) \cong(B: Z \rightarrow W))$ or $A \equiv B$ (respectively $A \cong B$ ) for short. 


\subsection{Hyperspace}

Denote the set of all compact subsets of a compact metric space $S$ by $\mathcal{K}(S)$ and endow $\mathcal{K}(S)$ with the Vietoris topology. Because $S$ is a metric space, the Vietoris topology is generated by the Hausdorff metric, and this metric makes $\mathcal{K}(S)$ into a compact Polish space. The reader can find more details about $\mathcal{K}(S)$ and its topology in [24, Section 4.F].

The next lemma is [11, Lemma 3.7], and it will play an important role in Section 5. Recall that a critical ingredient towards showing that a separable Banach space $X$ embeds isometrically into $C(\Delta)$ is the fact that $B_{X^{*}}$ is separable and metrisable in the weak* topology and thus an image of the Cantor set under some continuous map. The next lemma gives us a way to parametrise the selection of this continuous surjection, and it is our tool to bring abstract Banach spaces into a concrete isometric copy of it in $C(\Delta)$.

Lemma 2.4. Let $\Delta$ be the Cantor set. There exists a Borel function

$$
Q: \mathcal{K}(\Delta) \rightarrow C(\Delta, \Delta)
$$

such that, for each $K \in \mathcal{K}(\Delta), Q(K): \Delta \rightarrow \Delta$ is a continuous function onto $K$. Therefore, if $M$ is a compact metric space and $h: \Delta \rightarrow M$ is a continuous surjection, we have that

$$
H: K \in \mathcal{K}(M) \mapsto h \circ Q\left(h^{-1}(K)\right) \in C(\Delta, M)
$$

is a Borel function and, for each $K \in \mathcal{K}(M), H(K): \Delta \rightarrow M$ is a continuous function onto $K$.

\subsection{Trees}

For a set $\Lambda$, we let $\Lambda^{<\mathbb{N}}=\bigcup_{n \in \mathbb{N}} \Lambda^{n}$. If $\bar{n}=\left(n_{1}, \ldots, n_{k}\right) \in \Lambda^{<\mathbb{N}}$, we write $|\bar{n}|=k$, and $|\bar{n}|$ is called the length of $\bar{n}$. If $\ell \leqslant k$, we write $\bar{n} \uparrow \ell=\left(n_{1}, \ldots, n_{\ell}\right)$. Analogously, if $\bar{n}=\left(n_{j}\right)_{j=1}^{\infty} \in \Lambda^{\mathbb{N}}$ and $\ell \in \mathbb{N}$, we write $\bar{n} \uparrow \ell=\left(n_{1}, \ldots, n_{\ell}\right)$. Define an order $\leq$ on $\Lambda^{<\mathbb{N}}$ by setting $\bar{n} \leq \bar{m}$ if $\bar{n}$ is an initial segment of $\bar{m}$; that is, if $|\bar{n}| \leqslant|\bar{m}|$ and $\bar{m} \uparrow|\bar{n}|=\bar{n}$. If $\bar{m} \in \Lambda^{\mathbb{N}}$, we define $\bar{n} \leq \bar{m}$ analogously. A subset $T \subset \Lambda^{<\mathbb{N}}$ is called a tree on $\Lambda$ if for all $t \in T$ and all $s \in \Lambda^{<\mathbb{N}}, s \leq t$ implies $s \in T$. A tree $T$ is called pruned if for all $s \in T$ there exists $t \in T \backslash\{s\}$ with $s \leq t$.

If $T$ is a tree on a set $\Lambda$, an element $\beta \in \Lambda^{\mathbb{N}}$ is called a branch of $T$ if $\beta \uparrow k \in T$ for all $k \in \mathbb{N}$ and we denote the subset of $\Lambda^{\mathbb{N}}$ containing all branches of $T$ by [T]. We say that a tree $T$ on $\Lambda$ is ill-founded if $[T] \neq \emptyset$; otherwise, $T$ is called well-founded. If $\Lambda=\mathbb{N}$, we denote the set of all trees on $\mathbb{N}$ by $\operatorname{Tr}$ and the subsets of all ill-founded trees and well-founded trees by IF and WF, respectively.

\section{The Adjoint Map as a Borel Function}

The main result of this section is Theorem 3.1, which is a generalisation of [11, Theorem 1.1]. To prepare for the proof, we first introduce a coding for the unit ball of the duals of separable Banach spaces as compact subsets of the product space $[-1,1]^{\mathbb{N}}$ (we follow the approach of [16, Section 2.1.2]).

Given $X \in \mathrm{SB}$, define $K_{X^{*}} \subset[-1,1]^{\mathbb{N}}$ by $^{2}$

$$
K_{X^{*}}=\left\{x^{*} \in[-1,1]^{\mathbb{N}}: \exists f \in B_{X^{*}}, \forall n \in \mathbb{N}, x_{n}^{*}=\frac{f\left(d_{n}(X)\right)}{\left\|d_{n}(X)\right\|}\right\},
$$

where if $d_{n}(X)=0$, we let $x_{n}^{*}=0$ above. It is easy to see that the subset $\mathbb{D} \subset \mathrm{SB} \times[-1,1]^{\mathbb{N}}$ given by

$$
\left(X, x^{*}\right) \in \mathbb{D} \Leftrightarrow x^{*} \in K_{X^{*}}
$$

\footnotetext{
${ }^{2}$ As a rule, we denote elements of a space $X$ by $x$, functionals in the dual $X^{*}$ by $f$, elements in the product space $[-1,1]^{\mathbb{N}}$ by $x^{*}$ and their $n$th coordinate by $x_{n}^{*}$.
} 
is Borel and that $K_{X^{*}}$ is a compact subset of $[-1,1]^{\mathbb{N}}$ for all $X \in \mathrm{SB}$ (see [16, Section 2.1.2]). Therefore, by [24, Theorem 28.8], the map

$$
X \in \mathrm{SB} \mapsto K_{X^{*}} \in \mathcal{K}\left([-1,1]^{\mathbb{N}}\right)
$$

is Borel. Moreover, one can easily see that for each $X \in \mathrm{SB}$ there exists a linear isometry

$$
i_{X}: \overline{\operatorname{span}}\left\{K_{X^{*}}\right\} \subset \ell_{\infty} \rightarrow X^{*}
$$

such that

$$
f=i_{X}\left(x^{*}\right) \text { implies } x_{n}^{*}=\left\{\begin{array}{ll}
\frac{f\left(d_{n}(X)\right)}{\left\|d_{n}(X)\right\|}, & \text { if } d_{n}(X) \neq 0, \\
0, & \text { if } d_{n}(X)=0,
\end{array} \text { for all } n \in \mathbb{N}\right.
$$

(see [16, Section 2.1.2] or [11, Section 3]).

We will also need a Borel way of computing the functional evaluation of an element $x^{*} \in K_{X^{*}}$ at some $x \in X$. We proceed as follows. Consider the Borel set

$$
\mathbb{A}=\left\{\left(X, x, x^{*}\right) \in \mathrm{SB} \times C(\Delta) \times[-1,1]^{\mathbb{N}}: x \in X, x^{*} \in K_{X^{*}}\right\}
$$

and define a map $\alpha: \mathbb{A} \rightarrow \mathbb{R}$ by letting

$$
\alpha\left(X, x, x^{*}\right)=\left\langle i_{X}\left(x^{*}\right), x\right\rangle
$$

for all $\left(X, x, x^{*}\right) \in \mathbb{A}$, where, given $f \in X^{*}$ and $x \in X,\langle f, x\rangle$ denotes the functional evaluation of $f$ at $x$; that is, $f(x)$. By [11, Lemma 3.8], $\alpha$ is a Borel map.

We may now state the main result of this section.

Theorem 3.1. Let $\mathcal{B} \subset \mathcal{L}_{\mathrm{SD}}$ be a Borel subset. There exists a Borel assignment $A \in \mathcal{B} \mapsto A^{\bullet} \in \mathcal{L}$ - that is, an assignment $(X, Y, A) \in \mathcal{B} \mapsto\left(Y^{\bullet}, X^{\bullet}, A^{\bullet}\right) \in \mathcal{L}-$ such that $A^{\bullet}$ is isometric to $A^{*}$ for all $A \in \mathcal{B}$.

Moreover, the set

$$
\mathcal{C}=\left\{(X, Y, A, g, x) \in \mathcal{B} \times C(\Delta) \times C(\Delta): g \in Y^{\bullet}, x \in X\right\}
$$

is Borel and there exists a Borel map $[\cdot, \cdot \cdot]_{(\cdot, \cdot, \cdot)}: \mathcal{C} \rightarrow \mathbb{R}$ such that, for each $(X, Y, A) \in \mathcal{B}$,

1. $[\cdot, \cdot]_{(X, Y, A)}$ is bilinear and norm continuous and

2. $g \in Y^{\bullet} \mapsto[g, \cdot]_{(X, Y, A)} \in X^{*}$ is isometric to $A^{*}$.

Proof. Because $\mathcal{B}$ is Borel, the subsets

$$
\mathbb{A}_{1}=\left\{X \in \mathrm{SB}: \exists Y \in \mathrm{SB}, \exists \hat{A} \in C(\Delta)^{\mathbb{N}} \text { s.t. }(X, Y, \hat{A}) \in \mathcal{B}\right\}
$$

and

$$
\mathbb{A}_{2}=\left\{Y \in \mathrm{SB}: \exists X \in \mathrm{SB}, \exists \hat{A} \in C(\Delta)^{\mathbb{N}} \text { s.t. }(X, Y, \hat{A}) \in \mathcal{B}\right\}
$$

are analytic. Because $\mathbb{A}_{1} \cup \mathbb{A}_{2} \subset \mathrm{SD}$ and SD is coanalytic, Lusin's separation theorem [24, Theorem 14.7] gives a Borel subset $\mathbb{B} \subset \mathrm{SD}$ such that $\mathbb{A}_{1} \cup \mathbb{A}_{2} \subset \mathbb{B}$. Define

$$
\mathbb{E}=\left\{\left(X, x^{*}, y\right) \in \mathbb{B} \times[-1,1]^{\mathbb{N}} \times \Delta: x^{*} \in K_{X^{*}}\right\}
$$


and let $\gamma: \mathbb{E} \rightarrow \mathbb{R}$ be the Borel map given by [11, Corollary 3.10]. The properties of $\gamma$ that are important for our goals are

1. $\gamma\left(X, x^{*}, \cdot\right) \in C(\Delta)$ for all $X \in \mathbb{B}$ and all $x^{*} \in K_{X^{*}}$ and

2. $\left\|\gamma\left(X, x^{*}, \cdot\right)\right\|=\left\|x^{*}\right\|_{\infty}$ for all $X \in \mathbb{B}$ and all $x^{*} \in K_{X^{*}}$.

Given $X \in \mathbb{B}$ and $x^{*} \in K_{X^{*}}$, the reader should interpret $\gamma\left(X, x^{*}, \cdot\right)$ as the coding (back in the universal space $C(\Delta)$ ) of the functional of $X^{*}$ that is coded by $x^{*} \in K_{X^{*}}$.

For each $X \in \mathbb{B}$, define

$$
X^{\bullet}=\left\{g \in C(\Delta): \exists x^{*} \in K_{X^{*}}, \exists \lambda \in \mathbb{R}, \text { s.t. } \forall y \in \Delta, g(y)=\lambda \gamma\left(X, x^{*}, y\right),\right\} .
$$

It is shown in the proof of [11, Theorem 1.1] that the assignment $X \in \mathbb{B} \mapsto X^{\bullet} \in \mathrm{SB}$ is Borel and that $X^{\bullet}$ is linearly isometric to $X^{*}$ for all $X \in \mathbb{B}$. Moreover, let

$$
\mathcal{A}=\left\{(X, x, g) \in \mathbb{B} \times C(\Delta) \times C(\Delta): x \in X, g \in X^{\bullet}\right\}
$$

and for each $(X, x, g) \in \mathcal{A}$, with $g=\lambda \gamma\left(X, x^{*}, \cdot\right)$, define

$$
\langle g, x\rangle_{X}=\lambda \alpha\left(X, x, x^{*}\right) .
$$

Then $\mathcal{A}$ is Borel and it was proved in the proof of [11, Theorem 1.1] that $\langle g, x\rangle_{X}$ does not depend on the representative $\lambda \gamma\left(X, x^{*}, \cdot\right)$ of $g$. Finally, the proof of [11, Theorem 1.1] also shows that

$$
(X, x, g) \in \mathcal{A} \mapsto\langle g, x\rangle_{X} \in \mathbb{R}
$$

is Borel and that, for each $X \in \mathbb{B}$,

3. $\langle\cdot, \cdot\rangle_{X}$ is bilinear and norm continuous and

4. $g \in X^{\bullet} \mapsto\langle g, \cdot\rangle_{X} \in X^{*}$ is a surjective linear isometry.

We now return to the proof of the theorem. For that, we define an assignment $(X, Y, \hat{A}) \in \mathcal{B} \mapsto \hat{A}^{\bullet} \in$ $C(\Delta)^{\mathbb{N}}$ so that the sequence $\hat{A}^{\bullet} \in C(\Delta)^{\mathbb{N}}$ will work as the coding of the adjoint operator $A^{*}: Y^{*} \rightarrow X^{*}$ analogously as $\hat{A}$ codes the operator $A: X \rightarrow Y$. For each $n \in \mathbb{N}$, define a map $x_{n}^{*}: \mathcal{B} \rightarrow \mathbb{R}^{\mathbb{N}}$ by letting

$$
x_{n}^{*}(X, Y, \hat{A})=\left(\left\langle d_{n}\left(Y^{\bullet}\right), \frac{\hat{A}(m)}{\left\|d_{m}(X)\right\|}\right\rangle_{Y}\right)_{m} \in \mathbb{R}^{\mathbb{N}},
$$

where $\hat{A}(m) /\left\|d_{m}(X)\right\|$ is taken to be 0 above if $d_{m}(X)=0$. Because the maps $(X, Y, \hat{A}) \in \mathcal{B} \mapsto \hat{A}(m) \in$ $C(\Delta),(X, x, g) \in \mathcal{A} \mapsto\langle g, x\rangle_{X} \in \mathbb{R}$ and $X \in \mathrm{SB} \mapsto d_{m}(X)$ are Borel for all $m \in \mathbb{N}$, the map $x_{n}^{*}$ is a Borel map for all $n \in \mathbb{N}$.

For each $n \in \mathbb{N}$, define $\hat{A}^{\bullet}(n) \in C(\Delta)$ by letting $\hat{A}^{\bullet}(n)=0$ if $x_{n}^{*}(X, Y, \hat{A})=0$, and

$$
\hat{A}^{\bullet}(n)=\left\|x_{n}^{*}(X, Y, \hat{A})\right\| \cdot \gamma\left(X, \frac{x_{n}^{*}(X, Y, \hat{A})}{\left\|x_{n}^{*}(X, Y, \hat{A})\right\|}, \cdot\right)
$$

otherwise. By the definition of $X^{\bullet}, \hat{A}^{\bullet}(n) \in X^{\bullet}$ for all $n \in \mathbb{N}$.

Clearly, $(X, Y, \hat{A}, y) \in \mathcal{B} \times \Delta \mapsto \hat{A}^{\bullet}(n)(y) \in \mathbb{R}$ is Borel, which implies that $(X, Y, \hat{A}) \in \mathcal{B} \mapsto$ $\hat{A}^{\bullet}(n) \in C(\Delta)$ is Borel for all $n \in \mathbb{N}$. Therefore, $(X, Y, \hat{A}) \in \mathcal{B} \mapsto \hat{A}^{\bullet} \in C(\Delta)^{\mathbb{N}}$ is Borel. In particular, the assignment

$$
(X, Y, \hat{A}) \in \mathcal{B} \mapsto\left(Y^{\bullet}, X^{\bullet}, \hat{A}^{\bullet}\right) \in \mathrm{SB} \times \mathrm{SB} \times C(\Delta)^{\mathbb{N}}
$$

is Borel. 
Claim 3.2. For each $(X, Y, \hat{A}) \in \mathcal{B}$, we have that $\left(Y^{\bullet}, X^{\bullet}, \hat{A}^{\bullet}\right) \in \mathcal{L}$. In particular, the assignment $(X, Y, \hat{A}) \in \mathcal{B} \mapsto\left(Y^{\bullet}, X^{\bullet}, \hat{A}^{\bullet}\right) \in \mathcal{L}$ is well defined (and it is Borel).

Proof. Fix $(X, Y, \hat{A}) \in \mathcal{B}$. Let $q_{1}, \ldots, q_{k} \in \mathbb{Q}$ and $n_{1}, \ldots, n_{k} \in \mathbb{N}$. Because $g \in X^{\bullet} \mapsto\langle g, \cdot\rangle_{X} \in X^{*}$ is a surjective linear isometry and $\langle\cdot, \cdot\rangle_{X}$ is bilinear, we have that

$$
\begin{aligned}
\left\|\sum_{i=1}^{k} q_{i} \hat{A}^{\bullet}\left(n_{i}\right)\right\| & =\sup _{m \in \mathbb{N}}\left|\left\langle\sum_{i=1}^{k} q_{i} \hat{A}^{\bullet}\left(n_{i}\right), \frac{d_{m}(X)}{\left\|d_{m}(X)\right\|}\right\rangle_{X}\right|^{k} \\
& =\sup _{m \in \mathbb{N}}\left|\sum_{i=1}^{k} q_{i}\left\langle\hat{A}^{\bullet}\left(n_{i}\right), \frac{d_{m}(X)}{\left\|d_{m}(X)\right\|}\right\rangle_{X}\right| .
\end{aligned}
$$

By the definition of $\langle\cdot, \cdot\rangle_{X}$ and using the representative of $\hat{A}^{\bullet}\left(n_{i}\right)$ given in $(* *)$, this gives that

$$
\left\|\sum_{i=1}^{k} q_{i} \hat{A}^{\bullet}\left(n_{i}\right)\right\|=\sup _{m \in \mathbb{N}}\left|\sum_{i=1}^{k} q_{i}\left\|x_{n_{i}}^{*}(X, Y, \hat{A})\right\| \cdot \alpha\left(X, \frac{d_{m}(X)}{\left\|d_{m}(X)\right\|}, \frac{x_{n_{i}}^{*}(X, Y, \hat{A})}{\left\|x_{n_{i}}^{*}(X, Y, \hat{A})\right\|}\right)\right| .
$$

Finally, because $\alpha\left(X, x, x^{*}\right)=\left\langle i_{X}\left(x^{*}\right), x\right\rangle$, the property of $i_{X}$ given in $(*)$ implies that

$$
\begin{aligned}
\left\|\sum_{i=1}^{k} q_{i} \hat{A}^{\bullet}\left(n_{i}\right)\right\| & =\sup _{m \in \mathbb{N}}\left|\sum_{i=1}^{k} q_{i}\left\langle d_{n_{i}}\left(Y^{\bullet}\right), \frac{\hat{A}(m)}{\left\|d_{m}(X)\right\|}\right\rangle\right| \\
& =\sup _{m \in \mathbb{N}}\left|\left\langle\sum_{i=1}^{k} q_{i} d_{n_{i}}\left(Y^{\bullet}\right), \frac{\hat{A}(m)}{\left\|d_{m}(X)\right\|}\right\rangle\right| \\
& \leqslant \sup _{m \in \mathbb{N}}\left\|\frac{\hat{A}(m)}{\left\|d_{m}(X)\right\|}\right\| \cdot\left\|\sum_{i=1}^{k} q_{i} d_{n_{i}}\left(Y^{\bullet}\right)\right\| \\
& =\|A\| \cdot\left\|\sum_{i=1}^{k} q_{i} d_{n_{i}}\left(Y^{\bullet}\right)\right\| .
\end{aligned}
$$

Because $q_{1}, \ldots, q_{k} \in \mathbb{Q}$ and $n_{1}, \ldots, n_{k} \in \mathbb{N}$ are arbitrary, it follows that $\left(Y^{\bullet}, X^{\bullet}, \hat{A}^{\bullet}\right) \in \mathcal{L}$; that is, $\hat{A}^{\bullet}$ codes an operator $A^{\bullet}: Y^{\bullet} \rightarrow X^{\bullet}$.

Claim 3.3. The operator $A^{\bullet}: Y^{\bullet} \rightarrow X^{\bullet}$ is isometric to $A^{*}: Y^{*} \rightarrow X^{*}$ for all $(X, Y, \hat{A}) \in \mathcal{B}$.

Proof. For each $Z \in\{X, Y\}$, define $j_{Z}: Z^{\bullet} \rightarrow Z^{*}$ by letting $j_{Z}(g)=\langle g, \cdot\rangle_{Z} \in Z^{*}$ for all $g \in Z^{\bullet}$. Therefore, both $j_{X}$ and $j_{Y}$ are linear isometries. Let us show that $A^{*} \circ j_{Y}=j_{X} \circ A^{\bullet}$.

Fix $n \in \mathbb{N}$. If $d_{n}\left(Y^{\bullet}\right)=0$, then clearly $A^{*} \circ j_{Y}\left(d_{n}\left(Y^{\bullet}\right)\right)=j_{X} \circ A^{\bullet}\left(d_{n}\left(Y^{\bullet}\right)\right)$. Assume $d_{n}\left(Y^{\bullet}\right) \neq 0$. Given $m \in \mathbb{N}$, we have that

$$
A^{*} \circ j_{Y}\left(d_{n}\left(Y^{\bullet}\right)\right)\left(d_{m}(X)\right)=j_{Y}\left(d_{n}\left(Y^{\bullet}\right)\right)\left(A\left(d_{m}(X)\right)\right)=\left\langle d_{n}\left(Y^{\bullet}\right), \hat{A}(m)\right\rangle_{Y}
$$

By the definition of $x_{n}^{*}(X, Y, \hat{A})$ and the property of $i_{X}$ given in $(*)$, it follows that

$$
\left\langle i_{X}\left(x_{n}^{*}(X, Y, \hat{A})\right), d_{m}(X)\right\rangle=\left\langle d_{n}\left(Y^{\bullet}\right), \hat{A}(m)\right\rangle_{Y} .
$$


By the definition of $\alpha$ and $\gamma$, we conclude that

$$
\begin{aligned}
\left\langle i_{X}\left(x_{n}^{*}(X, Y, \hat{A})\right), d_{m}(X)\right\rangle & =\left\|x_{n}^{*}(X, Y, \hat{A})\right\| \cdot \alpha\left(X, d_{m}(X), \frac{x_{n}^{*}(X, Y, \hat{A})}{\left\|x_{n}^{*}(X, Y, \hat{A})\right\|}\right) \\
& =j_{X}\left(\left\|x_{n}^{*}(X, Y, \hat{A})\right\| \cdot \gamma\left(X, \frac{x_{n}^{*}(X, Y, \hat{A})}{\left\|x_{n}^{*}(X, Y, \hat{A})\right\|}, \cdot\right)\right)\left(d_{m}(X)\right) \\
& =\left(j_{X} \circ A^{\bullet}\left(d_{n}\left(Y^{\bullet}\right)\right)\right)\left(d_{m}(X)\right) .
\end{aligned}
$$

Because $\left(d_{m}(X)\right)_{m}$ is dense in $X$, it follows that

$$
A^{*} \circ j_{Y}\left(d_{n}\left(Y^{\bullet}\right)\right)=j_{X} \circ A^{\bullet}\left(d_{n}\left(Y^{\bullet}\right)\right)
$$

for all $n \in \mathbb{N}$, and we conclude that $A^{*} \circ j_{Y}=j_{X} \circ A^{\bullet}$.

This concludes the proof of the first part of the theorem. We now move to its second statement.

Claim 3.4. The assignment $(X, Y, \hat{A}) \in \mathcal{L} \mapsto\|A\| \in \mathbb{R}$ is Borel.

Proof. This follows because for each $n \in \mathbb{N}$ the assignment

$$
(X, Y, \hat{A}) \in \mathcal{L} \mapsto \frac{\hat{A}(n)}{\left\|d_{n}(X)\right\|} \in \mathbb{R},
$$

where $\hat{A}(n) /\left\|d_{n}(X)\right\|$ is taken to be 0 if $d_{n}(X)=0$, is Borel. Hence, because $\|A\|=\sup _{n} \hat{A}(n) /\left\|d_{n}(X)\right\|$, it follows that $(X, Y, \hat{A}) \in \mathcal{L} \mapsto\|A\| \in \mathbb{R}$ is Borel.

Claim 3.5. Let

$$
\mathcal{D}=\left\{(X, Y, \hat{A}, g) \in \mathcal{B} \times C(\Delta): g \in Y^{\bullet}\right\}
$$

Then $\mathcal{D}$ is Borel and the map

$$
(X, Y, \hat{A}, g) \in \mathcal{D} \mapsto A^{\bullet}(g) \in C(\Delta)
$$

is Borel.

Proof. Recall the definition of the operator $A^{\bullet}: Y^{\bullet} \rightarrow X^{\bullet}$ coded by $\hat{A}^{\bullet}$. Given $g \in Y^{\bullet}$ and a sequence $\left(n_{i}\right)_{i}$ of natural numbers such that $g=\lim _{i} d_{n_{i}}\left(Y^{\bullet}\right)$, we have $A^{\bullet}(g)=\lim _{i} \hat{A}^{\bullet}\left(n_{i}\right)$. Moreover, by the definition of $\mathcal{L}$ and because $\left\|A^{\bullet}\right\|=\|A\|$, we have that

$$
\left\|A^{\bullet}(g)-\hat{A}^{\bullet}(n)\right\| \leqslant\|A\| \cdot\left\|g-d_{n}\left(Y^{\bullet}\right)\right\|
$$

for all $n \in \mathbb{N}$.

Let $h \in C(\Delta)$ and $\varepsilon>0$. By the above, it follows that

$$
\begin{aligned}
\left\|A^{\bullet}(g)-h\right\| \leqslant \varepsilon \Leftrightarrow & \forall \delta \in(0, \varepsilon) \cap \mathbb{Q}, \exists n \in \mathbb{N} \text { s.t. } \\
& \left(\left\|g-d_{n}\left(Y^{\bullet}\right)\right\| \leqslant \frac{\delta}{\|A\|}\right) \wedge\left(\left\|\hat{A}^{\bullet}(n)-h\right\| \leqslant \varepsilon+\delta\right) .
\end{aligned}
$$

By Claim 3.4, the right-hand side of (1) gives us a Borel condition on $(X, Y, \hat{A}, g) \in \mathcal{D}$. Therefore, the claim follows.

Let

$$
\mathcal{C}=\left\{(X, Y, \hat{A}, g, x) \in \mathcal{B} \times C(\Delta) \times C(\Delta): g \in Y^{\bullet}, x \in X\right\} .
$$


Because $Y \in \mathcal{B} \mapsto Y^{\bullet}$ is Borel, $\mathcal{C}$ is Borel. Given $(X, Y, \hat{A}, g, x) \in \mathcal{C}$, define

$$
[g, x]_{(X, Y, \hat{A})}=\left\langle A^{\bullet}(g), x\right\rangle_{X}
$$

By Claim 3.5, $[\cdot, \cdot]_{(\cdot, \cdot, \cdot)}: \mathcal{C} \rightarrow \mathbb{R}$ is Borel.

Fix $(X, Y, \hat{A}) \in \mathcal{B}$. It is clear that $[\cdot, \cdot]_{(X, Y, \hat{A})}$ is bilinear and norm-continuous. Finally, because $\left[d_{n}\left(Y^{\bullet}\right), d_{m}(X)\right]_{(X, Y, \hat{A})}=\left\langle A^{\bullet}\left(d_{n}\left(Y^{\bullet}\right)\right), d_{m}(X)\right\rangle_{X}$, Claim 3.3 implies that

$$
A^{*} \circ j_{Y}\left(d_{n}\left(Y^{\bullet}\right)\right)\left(d_{m}(X)\right)=\left[d_{n}\left(Y^{\bullet}\right), d_{m}(X)\right]_{(X, Y, \hat{A})}
$$

for all $n, m \in \mathbb{N}$. Therefore, $\left(j_{Y}, \operatorname{Id}_{X^{*}}\right)$ is an isometry between $g \in Y^{\bullet} \mapsto[g, \cdot]_{(X, Y, \hat{A})} \in X^{*}$ and $A^{*}: Y^{*} \rightarrow X^{*}$. This finishes the proof of the theorem.

\subsection{Consequences of Theorem 3.1}

The next corollary of Theorem 3.1 is a generalisation of the main result of [17]. If $\mathcal{A} \subset \mathcal{L}_{\mathrm{SD}}$, we define its dual collection $\mathcal{A}^{*}$ as

$$
\mathcal{A}^{*}=\left\{B \in \mathcal{L}: \exists A \in \mathcal{A} \text { with } B \text { is isomorphic to } A^{*}\right\}
$$

Corollary 3.6. Let $\mathcal{A} \subset \mathcal{L}_{\mathrm{SD}}$ be analytic. Then the set $\mathcal{A}^{*}$ is analytic.

Proof. Let $\mathcal{A} \subset \mathcal{L}_{\mathrm{SD}}$ be analytic. Because $\mathcal{L}_{\mathrm{SD}}$ is coanalytic, Lusin's separation theorem implies that there is a Borel subset $\mathcal{B}$ of $\mathcal{L}_{\mathrm{SD}}$ with $\mathcal{A} \subset \mathcal{B}$. Theorem 3.1 yields a Borel assignment - call it $D: \mathcal{B} \rightarrow \mathcal{L}$, with $D(X, Y, A)=\left(Y^{\bullet}, X^{\bullet}, A^{\bullet}\right)$ - such that $A^{\bullet}$ is isometric to $A^{*}$ for each $A \in \mathcal{B}$. Because analyticity is preserved under Borel maps, $D(\mathcal{A})$ is analytic. Likewise, the isomorphic saturation of an analytic set is analytic [7, Lemma 5.17] and thus the following set is analytic:

$$
\{B \in \mathcal{L}: \exists C \in D(\mathcal{A}) \text { with } B \text { isomorphic to } C\} .
$$

Because the above set is equal to the $\mathcal{A}^{*}$ in the statement of the corollary, this concludes the proof.

We now describe another corollary of Theorem 3.1. As noticed by G. Godefroy in [20, Problem 5.2], the result [11, Theorem 1.1] implies that the equivalence class $\langle X\rangle$ of a separable reflexive Banach space is Borel if and only if the equivalence class of its dual $\left\langle X^{*}\right\rangle$ is Borel. Theorem 3.1 allows us to obtain the analogous result for bounded operators.

Before stating this corollary, we need some terminology. Precisely, given a coding for a bounded operator between separable Banach spaces $\mathcal{B} \subset \mathcal{L}$, we define the isomorphic saturation of $\mathcal{B}$, denoted by $\langle\mathcal{B}\rangle$, by

$$
\langle\mathcal{B}\rangle=\{(B: Z \rightarrow W) \in \mathcal{L}: \exists(A: X \rightarrow Y) \in \mathcal{B}, B \cong A\}
$$

If $\mathcal{B}$ is a set containing a single operator, say, $\mathcal{B}=\{A: X \rightarrow Y\}$, we simply write $\langle A: X \rightarrow Y\rangle$, or $\langle A\rangle$ for short.

Let us point out two straightforward facts about any collection $\mathcal{B} \subset \mathcal{L}$. Precisely, given such $\mathcal{B}$, we have that

1. if $\mathcal{B}$ is analytic, so is $\langle\mathcal{B}\rangle[7$, Lemma 5.17] and

2. if $\mathcal{B} \subset \mathcal{L}_{\mathrm{SD}}$, then $\mathcal{B}^{*}$ is closed under isomorphism; that is, $\mathcal{B}^{*}=\left\langle\mathcal{B}^{*}\right\rangle$.

Corollary 3.7. Let $\mathcal{B} \subset \mathcal{L}$ be a collection of operators between reflexive spaces. Then $\langle\mathcal{B}\rangle$ is Borel if and only if $\mathcal{B}^{*}$ is Borel. In particular, if $A: X \rightarrow Y$ is an operator between reflexive Banach spaces, then $\langle A\rangle$ is Borel if and only if $\left\langle A^{*}\right\rangle$ is Borel. 
Proof. For the forward direction, suppose $\langle\mathcal{B}\rangle$ is Borel. Let us first notice that $\mathcal{B}^{*}$ is analytic. For that, consider the Borel assignment $(X, Y, \hat{A}) \in\langle\mathcal{B}\rangle \mapsto\left(Y^{\bullet}, X^{\bullet}, \hat{A}^{\bullet}\right) \in \mathcal{L}$ given by Theorem 3.1. Then

$$
\mathcal{B}^{*}=\left\langle\left\{\left(Y^{\bullet}, X^{\bullet}, \hat{A}^{\bullet}\right) \in \mathcal{L}:(X, Y, \hat{A}) \in\langle\mathcal{B}\rangle\right\}\right\rangle .
$$

Because the assignment above is Borel, $\left\{\left(Y^{\bullet}, X^{\bullet}, \hat{A}^{\bullet}\right) \in \mathcal{L}:(X, Y, \hat{A}) \in\langle\mathcal{B}\rangle\right\}$ is analytic, which implies that $\mathcal{B}^{*}$ is analytic.

Because $\langle\mathcal{B}\rangle$ is a collection of operators between reflexive spaces, we have that $\mathcal{B}^{*} \subset \mathcal{L}_{\mathrm{SD}}$. Because $\mathcal{L}_{\mathrm{SD}}$ is coanalytic, Lusin's separation theorem allows us to pick a Borel $\mathcal{C} \subset \mathcal{L}_{\mathrm{SD}}$ so that $\mathcal{B}^{*} \subset \mathcal{C}$. From now on, let $\Phi:(X, Y, \hat{A}) \in \mathcal{C} \mapsto\left(Y^{\bullet}, X^{\bullet}, \hat{A}\right) \in \mathcal{L}$ be the Borel assignment given by Theorem 3.1. Because $\langle\mathcal{B}\rangle$ is a collection of operators between reflexive spaces, it follows that $\mathcal{B}^{*}=\Phi^{-1}(\langle\mathcal{B}\rangle)$. Hence, $\mathcal{B}^{*}$ is Borel because $\langle\mathcal{B}\rangle$ is Borel.

For the backwards direction, suppose now that $\mathcal{B}^{*}$ is Borel. By the definition of $\mathcal{B}^{*}$, we have that $\mathcal{B}^{*}=\left\langle\mathcal{B}^{*}\right\rangle$ and $\left(\mathcal{B}^{*}\right)^{*}=\langle\mathcal{B}\rangle$. Therefore, the result follows from the forward direction that we just proved.

\section{Factoring Weakly Compact Operators Through a Single Space}

We start this section introducing some definitions and terminology that will be used throughout. The next definition in particular is central to the main results of this article.

Definition 4.1. Let $\mathcal{J} \subset \mathcal{L}$ be a coding for some class of operators between separable Banach spaces and $\mathcal{P}$ be a class of Banach spaces. We say that $\mathcal{J}$ is strongly bounded with respect to $\mathcal{P}$ (respectively complementably strongly bounded with respect to $\mathcal{P}$ ) if for each analytic subset $\mathcal{A} \subset \mathcal{J}$ there exists $Z \in \mathcal{P}$ so that every operator in $\mathcal{A}$ factors through a subspace of $Z$ (respectively factors through $Z$ ).

The motivation for this terminology comes from the definition of (complementably) strongly bounded classes of separable Banach spaces, introduced by Argyros and Dodos in [3] (we refer the reader to Remark 4.5 for details). Notice that the weakly compact operators between separable Banach spaces are not complementably strongly bounded with respect to the reflexive spaces. Indeed, W.B. Johnson and A. Szankowski showed in [23, Theorem 2.5] that there is no separable Banach space through which every compact operator factors and it is routine to observe that the compact operators between separable Banach spaces are a Borel subset of $\mathcal{L}$.

Definition 4.2. Given subsets $\mathbb{A}, \mathbb{B} \subset \mathrm{SB}$, we define the following collection of operators:

$$
\mathcal{W}_{\mathbb{A}, \mathbb{B}}=\{(A: X \rightarrow Y) \in \mathcal{W}: X \in \mathbb{A} \text { and } Y \in \mathbb{B}\}
$$

If $\mathbb{A}$ (respectively $\mathbb{B}$ ) is the isomorphism class $\langle Z\rangle$ in $\mathrm{SB}$ of a certain separable Banach space $Z$, we simply write $\mathcal{W}_{Z, \mathbb{B}}\left(\right.$ respectively $\left.\mathcal{W}_{\mathbb{A}, Z}\right)$.

Let $\mathrm{SD}, \mathrm{sb}, \mathrm{sb}^{*}, \mathrm{ub}$ and $\mathrm{ub}^{*}$ denote the subsets of SB coding the separable Banach spaces (1) with separable dual, (2) with shrinking bases, (3) whose duals have shrinking bases, (4) with unconditional bases and (5) whose duals have unconditional bases, respectively.

We can now state the main results of this section.

Theorem 4.3 (Theorem B). The sets $\mathcal{W}_{\mathrm{SB}, \mathrm{ub}}, \mathcal{W}_{\mathrm{ub}^{*}, \mathrm{SD}}$ and $\mathcal{W}_{\mathrm{sb}^{*}, \mathrm{SD}}$ are complementably strongly bounded with respect to the class of separable reflexive Banach spaces admitting Schauder bases.

Moreover, for $\mathcal{W}_{\mathrm{SB}, L_{1}[0,1]}$, we obtain the following stronger result.

Theorem 4.4 (Theorem A). There exists a reflexive $Z \in \mathrm{SB}$ with a Schauder basis so that every $A \in \mathcal{W}_{\mathrm{SB}, L_{1}[0,1]}$ factors through $Z$. 
For the proof that $\mathcal{W}_{\mathrm{SB}, \mathrm{ub}}$ and $\mathcal{W}_{\mathrm{SB}, L_{1}[0,1]}$ are strongly bounded with respect to the class of separable reflexive spaces admitting Schauder bases (Theorem 4.9), we will not need any of the results in the previous sections. On the other hand, the same result for the collections $\mathcal{W}_{\mathrm{sb}^{*}, \mathrm{SD}}$ and $\mathcal{W}_{\mathrm{ub}}$, SD will make heavy use of the machinery in Section 3 (Theorem 4.11).

Remark 4.5. We take a moment to justify the terminology of Definition 4.1. As mentioned in the Introduction, the terminology was motivated by the definition of (complementably) strongly bounded classes of separable Banach spaces, introduced by Argyros and Dodos in [3]. Recall that a subset $\mathcal{B} \subset \mathrm{SB}$ is called strongly bounded (respectively complementably strongly bounded) if for each analytic subset $\mathcal{A} \subset \mathcal{B}$ there exists $Z \in \mathcal{B}$ so that every element of $\mathcal{A}$ is isomorphic to a (respectively complemented) subspace of $Z$. Hence, it easily follows that $\mathcal{B}$ is (respectively complementably) strongly bounded if and only if the collection $\{\text { Id }: X \rightarrow X\}_{X \in \mathcal{B}} \subset \mathcal{L}$ is (respectively complementably) strongly bounded with respect to $\mathcal{B}$.

Because the proofs of Theorems 4.3 and 4.4 make use of the DFJP interpolation scheme, we start this section recalling it (see [15] for more details). Let $X$ be a Banach space and $W \subset X$ be a convex, symmetric and bounded subset. For each $n \in \mathbb{N}$, define a norm $|\cdot|_{n}$ on $X$ by letting

$$
|x|_{n}=\inf \left\{\lambda>0: \frac{x}{\lambda} \in 2^{n} W+2^{-n} B_{X}\right\}
$$

for all $x \in X$; this is the Minkowski gauge norm on $X$ associated to $W$. Given $p \in(1, \infty)$, define

$$
\Delta_{p}(X, W)=\left\{x \in X: \sum_{n}|x|_{n}^{p}<\infty\right\}
$$

and let $\|\cdot\|_{p}$ be given by $\|x\|_{p}=\left(\sum_{n}|x|_{n}^{p}\right)^{1 / p}$ for all $x \in \Delta_{p}(X, W)$. This defines a complete norm on $\Delta_{p}(X, W)$, and the space $\left(\Delta_{p}(X, W),\|\cdot\|_{p}\right)$ is called the $p$-interpolation space of the pair $(X, W)$.

We will need the following lemma. ${ }^{3}$

Lemma 4.6. Let $X$ and $Y$ be Banach spaces and $A: X \rightarrow Y$ be a weakly compact operator. Suppose that $Y$ has either an unconditional basis or is isomorphic to $L_{1}[0,1]$, and let $\left(P_{n}\right)_{n}$ denote either the sequence of partial sum projections associated to the unconditional basis of $Y$ or to $\left(T\left(h_{n}\right)\right)$, where $\left(h_{n}\right)$ is the Haar basis of $L_{1}[0,1]$ and $T: L_{1}[0,1] \rightarrow Y$ is an isomorphism. Then

$$
W=\overline{\operatorname{conv}}\left(\bigcup_{n \in \mathbb{N}} P_{n}\left(A\left(B_{X}\right)\right)\right) ;
$$

that is, the closed convex hull of the union over $n \in \mathbb{N}$ of $P_{n}\left(A\left(B_{X}\right)\right)$, is weakly compact.

Proof. If $Y$ has an unconditional basis, the result follows straightforwardly from [19, Theorem 3.3]. ${ }^{4}$ Assume that $Y$ is isomorphic to $L_{1}[0,1]$; without loss of generality, we can assume that $Y=L_{1}[0,1]$. To see that $W$ is weakly compact we use the uniform integrability condition (see [2, Definition 5.2.2]). Recall that a bounded weakly closed subset $W^{\prime} \subset L_{1}[0,1]$ is weakly compact if and only if for all $\varepsilon>0$ there exists $M>0$ so that $W^{\prime} \subset M B_{L_{2}[0,1]}+\varepsilon B_{L_{1}[0,1]}$. This standard fact can be deduced from [2, Lemma 5.2.6 and Theorem 5.2.9]. Because the latter holds for $W$, we are done. Indeed, because $A$ is weakly compact, given any $\varepsilon>0$ there exists $M>0$ so that $A\left(B_{X}\right) \subset M B_{L_{2}[0,1]}+\varepsilon B_{L_{1}[0,1]}$. Because $B_{L_{2}[0,1]}$ and $B_{L_{1}[0,1]}$ are invariant under the Haar basis projections, we have that $P_{n}\left(A\left(B_{X}\right)\right) \subset$ $M B_{L_{2}[0,1]}+\varepsilon B_{L_{1}[0,1]}$ for all $n \in \mathbb{N}$, which implies $W \subset M B_{L_{2}[0,1]}+\varepsilon B_{L_{1}[0,1]}$.

\footnotetext{
${ }^{3}$ The proof of Lemma 4.6 was given to us by W.B. Johnson and we reproduce it here with his permission (see [21]).

${ }^{4}$ It is shown in [19, Theorem 3.3] that if $T: Z \rightarrow X$ is a weakly compact operator and $\left(e_{n}\right)_{n}$ is an 1-unconditional basis for $X$, then, thinking of $X$ as a Banach lattice, the closure of the convex hull of $\left\{x \in X: \exists z \in B_{Z},|x| \leqslant|T z|\right\}$ is weakly compact (cf. [1]).
} 
We now describe a construction that will be crucial for Theorem 4.9. Because this construction will also be used for the purposes of Section 5, we define the objects below in considerably more generality than we will need for Theorem 4.9.

Given a Polish space $S$, let $\mathcal{F}(S)$ denote the space of all closed subsets of $S$ endowed with the EffrosBorel structure; that is, the $\sigma$-algebra generated by

$$
\{\{F \in \mathcal{F}(S): U \cap F \neq \emptyset\}: U \subset S \text { is open }\} .
$$

Given $E \in \mathrm{SB}$, write

$$
\mathcal{F}_{0}(E)=\{F \in \mathcal{F}(E): F \text { is bounded, convex and symmetric }\} .
$$

Construction 4.7. Consider $E \in \mathrm{SB}, y_{1}, y_{2} \in E$, and a map $Y \in \mathrm{SB}(E) \mapsto\left(e_{n}^{Y}\right)_{n} \in E^{\mathbb{N}}$ so that $\left(e_{n}^{Y}\right)_{n}$ is a Schauder basis for $Y$ for all $Y \in \mathrm{SB}(E)$. For each $Y \in \mathrm{SB}(E)$, let $\left(P_{n}^{Y}\right)_{n}$ be the sequence of partial sum projections associated to $\left(e_{n}^{Y}\right)_{n}$.

For each $(Y, W) \in \mathrm{SB}(E) \times \mathcal{F}_{0}(E)$, let

1. $E_{W}=\overline{\operatorname{conv}}\left(W \cup\left\{y_{1}, y_{2}\right\}\right)$,

2. $W_{(Y, W)}^{m}=P_{m}^{Y}\left(E_{W}\right)$,

3. $\|\cdot\|_{(Y, W), m}$ be the Minkowski gauge norm on $E$ associated to $W_{(Y, W)}^{m}$,

4. $W_{(Y, W)}=\overline{\operatorname{conv}}\left(\cup_{m \in \mathbb{N}} W_{(Y, W)}^{m}\right)$,

5. $Z_{(Y, W)}=\Delta_{2}\left(E, W_{(Y, W)}\right)$ and

6. $\|\cdot\|_{(Y, W)}$ be the norm of $\Delta_{2}\left(E, W_{(Y, W)}\right)$.

By [15, Lemma 1, iii)], for all $(Y, W) \in \mathrm{SB}(E) \times \mathcal{F}_{0}(E)$ there exists a bounded injective operator $C_{(Y, W)}: Z_{(Y, W)} \rightarrow E$. Moreover, if the auxiliary vectors $y_{1}, y_{2} \in C(\Delta)$ are chosen so that $e_{n}^{Y} \in$ $\operatorname{span}\left(W_{(Y, W)}\right)$ for all $n \in \mathbb{N}$ and all $(Y, W) \in \operatorname{SB}(E) \times \mathcal{F}_{0}(E)$, then $e_{n}^{Y} \in \operatorname{Im}\left(C_{(Y, W)}\right)$. In this case, we define

7. $z_{n}^{(Y, W)}=C_{(Y, W)}^{-1}\left(e_{n}^{Y}\right)$ for each $n \in \mathbb{N}$ and $(Y, W) \in \mathrm{SB}(E) \times \mathcal{F}_{0}(E)$.

Then $\left(z_{n}^{(Y, W)}\right)_{n}$ is a basis for $Z_{(Y, W)}$ for all $(Y, W) \in \mathrm{SB}(E) \times \mathcal{F}_{0}(E)$ (see [15] or [9, Theorem 9]).

The next Lemma is the version of [9, Proposition 14] that we need for our goals.

Lemma 4.8. Let $E \in \mathrm{SB}$ be either a space with an unconditional basis or isomorphic to $L_{1}[0,1]$, and let $\mathcal{B} \subset \mathcal{L}$ be a Borel collection of weakly compact operators all of which have $E$ as their codomain. Then there exists reflexive $Z \in \mathrm{SB}$ with a Schauder basis so that every operator in $\mathcal{B}$ factors through $Z$. Moreover, for every $A=(X, E, \hat{A}) \in \mathcal{B}$, there exists a weakly compact, convex, symmetric and bounded set $W_{A}$ such that $A\left(B_{X}\right) \subset W_{A}$ and $\Delta_{2}\left(E, W_{A}\right)$ is isomorphic to a complemented subspace of $Z$.

Proof. This proof consists of noticing that the methods in [9] (mainly Proposition 14) together with Lemma 4.6 give us the desired result. Precisely, we only need to use the notation defined before the lemma so that we can use the results of [9].

Let $\left(e_{n}\right)_{n}$ be either the unconditional basis of $E$ or $e_{n}=T\left(h_{n}\right)$, where $\left(h_{n}\right)$ is the Haar basis of $L_{1}[0,1]$ and $T: L_{1}[0,1] \rightarrow E$ is an isormophism. Consider the objects defined in Construction 4.7 associated to $E$, the constant assignment $(Y, W) \in \mathrm{SB}(E) \times \mathcal{F}_{0}(E) \mapsto\left(e_{n}\right)_{n} \in E^{\mathbb{N}}$ and some $y_{1}, y_{2} \in E$ with $y_{1}=y_{0}$ and $y_{2}=-y_{0}$, where $y_{0}=\sum_{n} a_{n} e_{n} \in E$ so that $a_{n} \neq 0$ for all $n \in \mathbb{N}$. Then $y_{1}, y_{2}$ satisfy the special condition in Construction 4.7. For each $A=(X, E, \hat{A}) \in \mathcal{B}$, define $W_{A}=W_{\left(E, \overline{A\left(B_{X}\right)}\right)}$ and $Z_{A}=Z_{\left(E, \overline{A\left(B_{X}\right)}\right)}$. By Lemma 4.6, $W_{A}$ is weakly compact for all $A \in \mathcal{B}$, so $Z_{A}$ is reflexive for every such $A$. Moreover, by the proof of [9, Proposition 14], $\Delta_{2}\left(E, W_{A}\right)$ is isomorphic to a complemented subspace of $Z$.

Because the map $Y \in \mathrm{SB}(E) \mapsto\left(e_{n}\right)_{n} \in E^{\mathbb{N}}$ is Borel, the result now follows analogously to the proof of [9, Proposition 14]. 
We can now present the proof of half of Theorem 4.3. As mentioned at the beginning of this section, this half only makes use of results within this current section.

Theorem 4.9. Both $\mathcal{W}_{\mathrm{SB}, \mathrm{ub}}$ and $\mathcal{W}_{\mathrm{SB}, L_{1}[0,1]}$ are complementably strongly bounded with respect to the class of separable reflexive spaces admitting Schauder bases.

Proof. The proof of this theorem is an adaptation of [9, Theorem 25] with the extra ingredient of Lemma 4.8 and Pełczyński universal space.

We start proving the result for $\mathcal{W}_{\mathrm{SB}, \text { ub }}$, so let $\mathcal{A} \subset \mathcal{W}_{\mathrm{SB} \text {,ub }}$ be an analytic subset. Let $U$ be Pełczyński famous universal space for unconditional basis; that is, $U$ has an unconditional basis and every Banach space with an unconditional basis embeds into $U$ complementably [27, Corollary 1]. Define

$$
\mathcal{A}_{U}=\{(X, U, \hat{A}) \in \mathcal{L}: \exists(Z, W, \hat{B}) \in \mathcal{A}, X \equiv Z, \hat{A} \sim \hat{B}\}
$$

where $\hat{A} \sim \hat{B}$ stands for the sequence $\hat{A}$ is equivalent to the sequence $\hat{B}$; that is, there exists $M \geqslant 1$ such that for every $k \in \mathbb{N}$ and every $a_{0}, \ldots, a_{k} \in \mathbb{R}$ we have

$$
\frac{1}{M}\left\|\sum_{i=0}^{k} a_{i} \hat{A}(i)\right\| \leqslant\left\|\sum_{i=0}^{k} a_{i} \hat{B}(i)\right\| \leqslant M\left\|\sum_{i=0}^{k} a_{i} \hat{A}(i)\right\| .
$$

Because equivalence of sequences is a Borel relation in $C(\Delta) \times C(\Delta)$ and being isometric is an analytic relation in $\mathrm{SB} \times \mathrm{SB}$ (see [16, Section 2.1.1]), $\mathcal{A}_{U}$ is analytic. Define $\mathcal{W}_{U}=\mathcal{W} \cap\left(\mathrm{SB} \times\{U\} \times C(\Delta)^{\mathbb{N}}\right)$. Because $\mathcal{W}$ is coanalytic, so is $\mathcal{W}_{U}$. Hence, Lusin's separation theorem gives us a Borel $\mathcal{B}_{U} \subset \mathcal{W}_{U}$ so that $\mathcal{A}_{U} \subset \mathcal{B}_{U}$.

By Lemma 4.8, there exists a reflexive Banach space with a Schauder basis so that every $(X, U, \hat{A}) \in$ $\mathcal{B}_{U}$ factors through $Z$. Let us observe that every operator in $\mathcal{A}$ also factors through $Z$. Indeed, let $(X, Y, \hat{A}) \in \mathcal{A}$, and let $I_{Y}: Y \rightarrow U$ and $P_{Y}: U \rightarrow \operatorname{Im}\left(I_{Y}\right)$ be an isomorphic embedding and a bounded projection on $\operatorname{Im}\left(I_{Y}\right)$, respectively. Because $\hat{A} \sim \widehat{I_{Y} \circ A}, I_{Y} \circ A: X \rightarrow U$ belongs to $\mathcal{A}_{U} \subset \mathcal{B}_{U}$, so there are bounded operators $B: X \rightarrow Z$ and $C: Z \rightarrow U$ so that $I_{Y} \circ A=C \circ B$. Hence, $A$ factors through $Z$ because $A=\left(I_{Y}^{-1} \circ P_{Y} \circ C\right) \circ B$.

The result for $\mathcal{W}_{\mathrm{SB}, L_{1}[0,1]}$ is analogous but simpler because the operators already have isomorphic codomains.

Before providing the proof for the second half of Theorem 4.3, we need the following lemma, which is a consequence of [15, Lemma 1].

Lemma 4.10. Let $X$ and $Y$ be dual spaces, $T: Y \rightarrow X$ be a weak ${ }^{*}$-to-weak ${ }^{*}$ continuous weakly compact operator and $W \subset X$ be a weakly compact, symmetric, convex and bounded subset so that $T\left(B_{Y}\right) \subset W$. Let $\Delta_{2}(X, W)$ be the 2-interpolation space of the pair $(X, W)$ and $J: \Delta_{2}(X, W) \rightarrow X$ be the standard inclusion. Then $T$ factors through $\Delta_{2}(X, W)$ and both $J$ and $J^{-1} \circ T$ are weak ${ }^{*}$-to-weak ${ }^{*}$ continuous.

Proof. Let $Z=\Delta_{2}(X, W)$. Because $T\left(B_{Y}\right) \subset W, J^{-1} \circ T: Y \rightarrow Z$ is well defined and bounded, $T$ factors through $Z$, because $T=J \circ J^{-1} \circ T$. Because $W$ is a weakly compact set, [15, Lemma 1, (iv)] implies that $Z$ is reflexive. Therefore, $J$ is weak ${ }^{*}$-to-weak continuous.

It is only left to show that $J^{-1} \circ T$ is weak*-to-weak* continuous. For that, let $\left(y_{i}\right)_{i \in I}$ be a weak* null net in $Y$. Let $Y_{*}$ and $X_{*}$ denote the preduals of $Y$ and $X$, respectively. Because $J: Y \rightarrow X$ is weak ${ }^{*}$-toweak* continuous, there exists a bounded map $J_{*}: X_{*} \rightarrow Y_{*}$ such that $J=\left(J_{*}\right)^{*}$. Because $J$ is injective, it follows from Hahn-Banach that $J_{*}$ has dense range. Hence, in order to show that $\left(J^{-1} \circ T\left(y_{i}\right)\right)_{i \in I}$ is a weak* null net, it is enough to notice the following. Let $x_{*} \in X_{*}$. Then

$$
\left(J^{-1} \circ T\left(y_{i}\right)\right)\left(J_{*}\left(x_{*}\right)\right)=\left(\left(J_{*}\right)^{*} \circ J^{-1} \circ T\left(y_{i}\right)\right)\left(x_{*}\right)=T\left(y_{i}\right)\left(x_{*}\right) .
$$


Because $T$ is weak ${ }^{*}$-to-weak* continuous, the net $\left(J^{-1} \circ T\left(y_{i}\right)\left(J_{*}\left(x_{*}\right)\right)\right)_{i \in I}$ converges to zero. Therefore, $J^{-1} \circ T$ is weak* ${ }^{*}$-to-weak* continuous.

Theorem 4.11. Both $\mathcal{W}_{\mathrm{ub}^{*}, \mathrm{SD}}$ and $\mathcal{W}_{\mathrm{sb}^{*}, \mathrm{SD}}$ are complementably strongly bounded with respect to the class of separable reflexive spaces admitting Schauder bases.

Proof. We first prove the statement for $\mathcal{W}_{\mathrm{sb}^{*}, \mathrm{SD}}$. For that, let $\mathcal{A} \subset \mathcal{W}_{\mathrm{sb}^{*}, \mathrm{SD}}$ be an analytic subset. Because $\mathcal{W}$ is coanalytic [9, Proposition 22], so is $\mathcal{W}_{\mathrm{SD}}$. Hence, Lusin's theorem [24, Theorem 18.1] gives a Borel subset $\mathcal{B} \subset \mathcal{W}_{\text {SD }}$ such that $\mathcal{A} \subset \mathcal{B}$. Let

$$
(X, Y, \hat{A}) \in \mathcal{B} \mapsto\left(Y^{\bullet}, X^{\bullet}, \hat{A}^{\bullet}\right) \in \mathcal{L}
$$

(or $A \in \mathcal{B} \mapsto A^{\bullet} \in \mathcal{L}$ for short) be the Borel assignment given by Theorem 3.1. Then the set

$$
\mathcal{A}^{\bullet}=\left\{\left(Y^{\bullet}, X^{\bullet}, \hat{A}^{\bullet}\right):(X, Y, \hat{A}) \in \mathcal{A}\right\} ;
$$

that is, the image of $\mathcal{A}$ under this assignment, is analytic. Because $X^{\bullet}$ has a shrinking basis for all $\left(Y^{\bullet}, X^{\bullet}, \hat{A}^{\bullet}\right) \in \mathcal{A}^{\bullet},[9$, Theorem 25] gives a reflexive Banach space $Z$ with a Schauder basis such that every $A^{\bullet} \in \mathcal{A}^{\bullet}$ factors through $Z$. Moreover, for every $A^{\bullet}: Y^{\bullet} \rightarrow X^{\bullet}$ in $\mathcal{A}^{\bullet}$ there exists a weakly compact, convex, symmetric and bounded subset $W_{A} \subset X^{\bullet}$ such that $A^{\bullet}\left(B\left(Y^{\bullet}\right)\right) \subset W_{A}$ and $\Delta_{2}\left(X^{\bullet}, W_{A}\right)$ is isomorphic to a complemented subspace of $Z$ (this follows directly from the proof of [9, Theorem 25]). For each such $A^{\bullet} \in \mathcal{A}^{\bullet}$, let $J_{A}: \Delta_{2}\left(X^{\bullet}, W_{A}\right) \rightarrow X^{\bullet}$ be the standard inclusion.

By Theorem 3.1, we can identify $X^{*}, Y^{*}$ and $A^{*}$ with $X^{\bullet}, Y^{\bullet}$ and $A^{\bullet}$, respectively. By Lemma 4.10, $J_{A}$ and $J_{A}^{-1} A^{\bullet}$ are weak*-to-weak* continuous. Hence, there exist maps $U_{A}: X \rightarrow \Delta_{2}\left(X^{\bullet}, W_{A}\right)_{*}$ and $V_{A}: \Delta_{2}\left(X^{\bullet}, W_{A}\right)_{*} \rightarrow Y$ such that $U_{A}^{*}=J_{A}$ and $V_{A}^{*}=J_{A}^{-1} A^{\bullet}$, where $\Delta_{2}\left(X^{\bullet}, W_{A}\right)_{*}$ denotes the predual of $\Delta_{2}\left(X^{\bullet}, W_{A}\right)$. This gives us that

$$
\left(V_{A} U_{A}\right)^{*}=U_{A}^{*} V_{A}^{*}=J_{A} J_{A}^{-1} A^{\bullet}=A^{\bullet}=A^{*} .
$$

Therefore, $V_{A} U_{A}=A$. This gives us that every $A \in \mathcal{A}$ factors through the predual of $Z$, and we are done.

In order to show that $\mathcal{W}_{\mathrm{ub}^{*}, \mathrm{SD}}$ is strongly bounded with respect to the class of separable reflexive Banach spaces with a shrinking basis, we only need to proceed exactly as for $\mathcal{W}_{\mathrm{sb}^{*}, \mathrm{SD}}$ but using Theorem 4.9 instead of [9, Theorem 25]. We leave this task to the reader.

Proof of Theorem 4.3. This follows from Theorem 4.9 and Theorem 4.11.

Our penultimate result of this subsection, Theorem 4.4, is an application of Theorem 4.9. But first we need the following proposition.

Proposition 4.12. The subset $\mathcal{W}_{\mathrm{SB}, L_{1}[0,1]}$ is analytic.

Proof. We start by showing that $\left\{(X, Y, \hat{A}) \in \mathcal{W}: Y=L_{1}[0,1]\right\}$ is analytic. For each $n \in \mathbb{N}$ and each $f \in L_{1}[0,1]$, let $f_{n} \in L_{1}[0,1]$ be a function that coincides with $f$ in $f^{-1}([-n, n])$, equals $n$ in $f^{-1}([n, \infty))$ and equals $-n$ in $f^{-1}((-\infty,-n])$. Then the map $f \in L_{1}[0,1] \rightarrow f_{n}^{2} \in L_{1}$ is clearly well defined and continuous. Because

$$
B_{L_{2}[0,1]}=\bigcap_{n \in \mathbb{N}}\left\{f \in L_{1}[0,1]: \int f_{n}^{2} d \mu \leqslant 1\right\},
$$

it follows that $B_{L_{2}[0,1]}$ is a closed subset of $L_{1}[0,1]$.

In order to conclude the proof, we use once again that a bounded subset $W \subset L_{1}[0,1]$ is weakly compact if and only if for all $\varepsilon>0$ there exists $M>0$ so that $W \subset M B_{L_{2}[0,1]}+\varepsilon B_{L_{1}[0,1]}$ (cf. [2, Lemma 5.2.6 and Theorem 5.2.9]). Fix closed linear subspaces $E, F \subset C(\Delta)$ with $E \equiv L_{1}[0,1]$ and $F \equiv L_{2}[0,1]$. Without loss of generality, we may assume that $B_{F}$ is a closed subset of $E$. 
Given $M, \varepsilon>0$, define a Borel map $S_{M, \varepsilon}:(x, y) \in E \times E \rightarrow M x+\varepsilon y \in E$. Then, and letting $\mathcal{C}=\{(X, Y, \hat{A}) \in \mathcal{W}: Y=E\}$, we have that

$$
\begin{aligned}
&(X, E, \hat{A}) \in \mathcal{C} \Leftrightarrow \forall \varepsilon \in \mathbb{Q}_{>0}, \exists M \in \mathbb{Q}_{>0}, \forall n \in \mathbb{N} \\
& \hat{A}(n)=0 \vee \frac{\hat{A}(n)}{\|\hat{A}(n)\|} \in S_{M, \varepsilon}\left(B_{F} \times B_{E}\right) .
\end{aligned}
$$

This is an analytic condition, so $\{(X, Y, \hat{A}) \in \mathcal{W}: Y=E\}$ is analytic.

We will now observe that $\mathcal{W}_{\mathrm{SB}, L_{1}[0,1]}$ is analytic. For that, fix a Schauder basis $\left(e_{j}\right)_{j}$ for $E$ and given a basic sequence $\left(z_{j}\right)_{j}$ in $C(\Delta)$ so that $\left(z_{j}\right)_{j} \sim\left(e_{j}\right)_{j}$, let $I_{\left(z_{j}\right)_{j}}: \overline{\operatorname{span}}\left\{z_{j}: j \in \mathbb{N}\right\} \rightarrow E$ be the isomorphism given by $z_{j} \mapsto e_{j}$. Then we only need to notice that

$$
\begin{aligned}
(X, Y, \hat{A}) \in \mathcal{W}_{\mathrm{SB}, L_{1}[0,1] \Leftrightarrow} & \exists\left(z_{j}\right)_{j} \in Y^{\mathbb{N}}\left(\left(z_{j}\right)_{j} \text { is a Schauder basis for } Y\right) \\
& \wedge\left(\left(z_{j}\right)_{j} \sim\left(e_{j}\right)_{j}\right) \wedge\left(\left(X, E,\left(I_{\left(z_{j}\right)_{j}}(\hat{A}(n))\right)_{n}\right) \in \mathcal{C}\right) .
\end{aligned}
$$

Indeed, the conditions ' $\left(z_{j}\right)_{j}$ is a Schauder basis for $Y^{\prime}$ ' and ' $\left(z_{j}\right)_{j} \sim\left(e_{j}\right)_{j}$ ' are clearly Borel conditions. Moreover, let

$$
\begin{aligned}
& \mathcal{D}=\left\{\left(Y,\left(z_{j}\right)_{j}, z\right) \in \mathrm{SB} \times C(\Delta)^{\mathbb{N}} \times C(\Delta): z \in Y\right. \text { and } \\
& \left.\qquad\left(z_{j}\right)_{j} \text { is a Schauder basis for } Y \text { and }\left(z_{j}\right)_{j} \sim\left(e_{j}\right)_{j}\right\},
\end{aligned}
$$

so the assignment $\left(Y,\left(z_{j}\right)_{j}, z\right) \in \mathcal{D} \mapsto I_{\left(z_{j}\right)_{j}}(z) \in E$ is Borel (cf. [11, Lemma 4.8]). This shows that $\mathcal{W}_{\mathrm{SB}, L_{1}[0,1]}$ is analytic.

Proof of Theorem 4.4. This follows from Theorem 4.9 and Proposition 4.12.

We conclude this section with the following proposition regarding the complexities of the other classes we are considering.

Proposition 4.13. The subsets $\mathcal{W}_{\mathrm{SD}, \mathrm{sb}}, \mathcal{W}_{\mathrm{sb}^{*}, \mathrm{SD}}, \mathcal{W}_{\mathrm{SB}, \mathrm{ub}}$ and $\mathcal{W}_{\mathrm{ub}^{*}, \mathrm{SD}}$ are not analytic.

Proof. The proof consists of citing work from previous papers. Let $\mathrm{REFL}_{\mathrm{b}}$ be the coding for the reflexive Banach spaces with a Schauder basis. By [10, Corollary 3.3], there exists a Borel reduction $\varphi: \operatorname{Tr} \rightarrow \mathrm{SB}$ of the set of well-founded trees WF to REFL ; that is, $\varphi^{-1}\left(\mathrm{REFL}_{\mathrm{b}}\right)=\mathrm{WF}$. Moreover, $\varphi(T)$ has a Schauder basis for all $T \in \mathrm{Tr}$. Because $\varphi(T) \in \mathrm{REFL}_{\mathrm{b}}$ for $T \in \mathrm{WF}$, we have that $\varphi(T)$ belongs to $\mathrm{SD}$ and $\mathrm{sb}$ for all $T \in \mathrm{WF}$. Moreover, because the dual of a reflexive space is reflexive and because if $X^{*}$ has a Schauder basis so does $X$, we also have that $\varphi(T) \in \mathrm{sb}^{*}$ for all $T \in$ WF. Because each $\varphi(T)$ has a basis, we have that $\varphi(T)$ is not reflexive for all $T \in \mathrm{IF}$. Therefore, the map $\Phi: \operatorname{Tr} \rightarrow \mathcal{L}$ given by

$$
\Phi(T)=\left(\varphi(T), \varphi(T),\left(d_{n}(\varphi(T))\right)_{n}\right) \in \mathcal{L}
$$

is a Borel reduction of WF to both $\mathcal{W}_{\mathrm{SD}, \mathrm{sb}}$ and $\mathcal{W}_{\mathrm{sb}^{*}, \mathrm{SD}}$. In particular, both $\mathcal{W}_{\mathrm{SD} \text {,sb }}$ and $\mathcal{W}_{\mathrm{sb}^{*}, \mathrm{SD}}$ are not analytic.

Now we consider the cases $\mathcal{W}_{\mathrm{SB}, \mathrm{ub}}$ and $\mathcal{W}_{\mathrm{ub}}{ }^{*}$,SD . In [4, Proposition 8.6] the authors show that subsets $\mathfrak{M} \mathfrak{C}^{1} \cap \mathcal{L}$ and $\mathfrak{S M} \mathfrak{M}_{\infty}^{1} \cap \mathcal{L}$ of $\mathcal{L}$ are $\Pi_{1}^{1}$-complete. The set $\mathfrak{M} \mathfrak{C}^{1}$ is the $\mathcal{S}_{1}$-weakly compact operators (a subset of weakly compact operators, defined in [8]) and the set $\mathfrak{S} \mathfrak{M}_{\infty}^{1}$ denotes the operators not preserving a $c_{0}$ spreading model. In the proof of [4, Proposition 8.6], in order to show that the respective sets are not analytic, the authors define Borel maps $f: \operatorname{Tr} \rightarrow \mathcal{L}$ and $g: \operatorname{Tr} \rightarrow \mathcal{L}$ with $f^{-1}\left(\mathfrak{M S}^{1} \cap \mathcal{L}\right)=$ WF and $g^{-1}\left(\mathfrak{S M}_{\infty}^{1} \cap \mathcal{L}\right)=$ WF. In the current proof, we simply note that, by definition, for each $T \in \operatorname{Tr}$, the domain space of the operator $f(T)$ is isometric to $\ell_{1}$ and the range space, denoted $Z$, has an unconditional basis. Furthermore, $T \in \mathrm{WF}$ if and only if $f(T) \in \mathcal{W}$. Therefore, $f^{-1}\left(\mathcal{W}_{\mathrm{SB}, \mathrm{ub}}\right)=\mathrm{WF}$. Therefore, $\mathcal{W}_{\mathrm{SB}, \mathrm{ub}}$ is not analytic. Moreover, the operator $g(T)$ is defined isometric to the preadjoint of the operator $f(T)$. Consequently, for each $T \in \operatorname{Tr}$, the domain space of $g(T)$ isometric to the predual of $Z$ and range is 
isometric to $c_{0}$. In addition, $T \in \operatorname{Tr}$ if and only if $g(T) \in \mathcal{W}$. Consequently, $g^{-1}\left(\mathcal{W}_{u b^{*}, \mathrm{SD}}\right)=\mathrm{WF}$. This completes the proof.

\section{Factoring Weakly Compact Operators Through Subspaces of a Single Space}

The main results of this section are Theorems 5.1 and Theorem 5.5. Recall that if $S$ is a standard Borel space, then $\sigma_{S}\left(\Sigma_{1}^{1}\right)$ denotes the $\sigma$-algebra on $S$ generated by the analytic subsets of $S$. A map $S \rightarrow M$ between standard Borel spaces is called $\sigma\left(\Sigma_{1}^{1}\right)$-measurable if the preimage of every Borel subset of $M$ is in $\sigma_{S}\left(\Sigma_{1}^{1}\right)$.

The second named author and R. Causey proved in [7, Theorem 5.8] that $\mathcal{W}$ is strongly bounded with respect to the class of separable Banach spaces. The next result gives us that $\mathcal{W}_{\mathrm{SB}, \mathrm{SD}}$ satisfies an even stronger property. Not only is $\mathcal{W}_{\mathrm{SB}, \mathrm{SD}}$ strongly bounded but the choice of the space through which the operators will factor can be done in a $\sigma\left(\Sigma_{1}^{1}\right)$-measurable way.

Theorem 5.1. Let $\mathcal{B} \subset \mathcal{W}_{\mathrm{SB}, \mathrm{SD}}$ be a Borel subset. Then there is a reflexive space $Z \in \mathrm{SB}$ and a $\sigma\left(\Sigma_{1}^{1}\right)$ measurable $\Psi: \mathcal{B} \rightarrow \mathrm{SB}(Z)$ so that each $A=(X, Y, \hat{A}) \in \mathcal{B}$ factors through $\Psi(A)$.

Moreover, setting

$$
\mathcal{D}=\{(X, Y, \hat{A}, x) \in \mathcal{B} \times C(\Delta): x \in X\},
$$

there exists a $\sigma\left(\Sigma_{1}^{1}\right)$-measurable map $\Phi: \mathcal{D} \rightarrow Z$ so that, letting $\Phi_{A}=\Phi(X, Y, \hat{A}, \cdot)$, we have that, for each $A=(X, Y, \hat{A}) \in \mathcal{B}$,

1. $\Phi_{A}(X)=\Psi(A)$,

2. $\Phi_{A}: X \rightarrow Z$ is a bounded linear map with norm at most 1 and

3. there exists a bounded operator $L: \Psi(A) \rightarrow Y$ so that $A=L \circ \Phi_{A} \cdot{ }^{5}$

The proof of Theorem 5.1 will take this entire section. We start setting some notation. Precisely, given a Borel $\mathbb{B} \subset \mathrm{SD}$, we will need to evoke a construction of a Borel map $Y \in \mathbb{B} \mapsto\left(e_{n}^{Y}\right)_{n} \in C(\Delta)^{\mathbb{N}}$ given by B. Bossard. Because this construction is rather technical, we chose to simply present here the properties of it that are necessary for our settings and refer the reader to the appropriate sources.

Precisely, B. Bossard showed that there exists a Borel map (see [16, Claim 5.21])

$$
Y \in \mathbb{B} \mapsto\left(e_{n}^{Y}\right)_{n} \in C(\Delta)^{\mathbb{N}}
$$

with the following properties:

1. $\left(e_{n}^{Y}\right)_{n}$ is a monotone Schauder basis of $C(\Delta)$ for all $Y \in \mathbb{B}[16$, Claim 5.13].

Considering the objects in Construction 4.7 associated to $C(\Delta)$, the (partial) assignment $Y \in \mathbb{B} \mapsto$ $\left(e_{n}^{Y}\right)_{n} \in C(\Delta)^{\mathbb{N}}$ and $1, y \in C(\Delta)$, where $y$ is any normalised function that separates points of $\Delta$, it holds that

2. $B_{Y} \subset W_{\left(Y, B_{Y}\right)}$ for all $Y \in \mathbb{B}$, in particular, by [26, Lemma 4], $Y$ isometrically embeds into $Z_{\left(Y, B_{Y}\right)}$.

3. $\left(z_{n}^{\left(Y, B_{Y}\right)}\right)_{n}$ is a monotone shrinking basis for $Z_{\left(Y, B_{Y}\right)}$ for all $Y \in \mathbb{B}$ [16, Page 83].

4. $Z_{\left(Y, B_{Y}\right)}$ is reflexive for all reflexive $Y \in \mathbb{B}[16$, Lemma 5.18].

5. $(Y, z) \in \mathbb{B} \times C(\Delta) \mapsto\|z\|_{\left(Y, B_{Y}\right), m} \in \mathbb{R}$ is Borel for all $m \in \mathbb{N}[16$, Claim 5.23].

However, the proofs of the properties above actually give us something stronger. In a nutshell, the unit ball $B_{Y}$ can be replaced by any closed, bounded, convex, symmetric subset of $Y$. Precisely, the assignment $(*)$ has the following stronger properties:

2'. $W \subset W_{(Y, W)}$ for all $(Y, W) \in \mathbb{B} \times \mathcal{F}_{0}(C(\Delta))$,

3'. $\left(z_{n}^{(Y, W)}\right)_{n}$ is a monotone shrinking basis for $Z_{(Y, W)}$ for all $(Y, W) \in \mathbb{B} \times \mathcal{F}_{0}(C(\Delta))$ and

${ }^{5}$ See Remark 5.12 for a more precise bound for $\|L\|$. 
4'. $Z_{(Y, W)}$ is reflexive if $W$ is weakly compact.

Moreover, arguments analogous to those in [16, Claim 5.22 and Claim 5.23] give us that

5'. $(Y, W, z) \in \mathbb{B} \times \mathcal{F}_{0}(C(\Delta)) \times C(\Delta) \mapsto\|z\|_{(Y, W), m} \in \mathbb{R}$ is Borel for all $m \in \mathbb{N}$.

The references for the stronger properties above are precisely the same as the references for the validity of their weaker versions.

\subsection{Factoring a Borel $\mathcal{B} \subset \mathcal{W}_{\mathrm{SB}, \mathrm{SD}}$ Through a Family of Reflexive Spaces with Bases}

Let $\mathcal{B} \subset \mathcal{W}_{\mathrm{SB}, \mathrm{SD}}$ be a Borel subset. By Lusin's separation theorem, there exists a Borel $\mathbb{B} \subset \mathrm{SD}$ containing all of the codomains of the operators in $\mathcal{B}$. Let

$$
Y \in \mathbb{B} \mapsto\left(e_{n}^{Y}\right)_{n} \in C(\Delta)^{\mathbb{N}}
$$

be the map $(*)$ above associated to $\mathbb{B}$. Moreover, consider the objects in Construction 4.7 associated to $C(\Delta)$, the assignment $Y \in \mathbb{B} \mapsto\left(e_{n}^{Y}\right)_{n} \in C(\Delta)^{\mathbb{N}}$ and $1, y \in C(\Delta)$, where $y$ is a normalised function that separates the points of $\Delta$. In order to simplify notation, for each $A=(X, Y, \hat{A}) \in \mathcal{B}$ and $n \in \mathbb{N}$, we write

$$
Z_{A}=Z_{\left(Y, \overline{A\left(B_{X}\right)}\right)},\|\cdot\|_{A}=\|\cdot\|_{\left(Y, \overline{A\left(B_{X}\right)}\right)}, W_{A}=W_{\left(Y, \overline{A\left(B_{X}\right)}\right)} \text { and } z_{n}^{A}=z_{n}^{\left(Y, \overline{A\left(B_{X}\right)}\right)} .
$$

It is straightforward to check that the map

$$
(X, Y, \hat{A}) \in \mathcal{B} \mapsto\left(Y, \overline{A\left(B_{X}\right)}\right) \in \mathbb{B} \times \mathcal{F}_{0}(C(\Delta))
$$

is Borel; therefore, by 5' above, the map $(A, z) \in \mathcal{B} \times C(\Delta) \mapsto\|z\|_{A, m} \in \mathbb{R}$ is Borel for all $m \in \mathbb{N}$. Therefore, $(A, z) \in \mathcal{B} \times C(\Delta) \mapsto\|z\|_{A}$ must be Borel. Hence, after $\|\cdot\|_{A}$-normalising, we can (and do) assume that

$\circ\left(z_{n}^{A}\right)_{n}$ is a normalised monotone shrinking basis for $Z_{A}$ for all $A \in \mathcal{B}$.

We now code the unit balls of the dual spaces $Z_{A}^{*}$. This will be done slightly differently than in Section 3. For this different coding, we fix an enumeration $\left(\alpha_{k}\right)_{k}$ of $\mathbb{Q}^{<\mathbb{N}}$ and write $\alpha_{k} \times\left(e_{n}^{Y}\right)$ in order to abbreviate $\sum_{j=1}^{m} a_{j} e_{j}^{Y}$, where $\alpha_{k}=\left(a_{1}, \ldots, a_{m}\right)$.

Given $A=(X, Y, \hat{A}) \in \mathcal{B}$, let

$$
K_{A}=\left\{w^{*} \in B_{\ell_{\infty}}: \exists f \in B_{Z_{A}^{*}}, \forall k \in \mathbb{N}, w_{k}^{*}=\frac{f\left(\alpha_{k} \times\left(z_{n}^{A}\right)\right)}{\left\|\alpha_{k} \times\left(z_{n}^{A}\right)\right\|_{A}}\right\} .
$$

Therefore, $K_{A} \in \mathcal{K}\left(B_{\ell_{\infty}}\right)$. Because $\left(\alpha_{k} \times\left(z_{n}^{A}\right)\right)_{k}$ is dense in $Z_{A}, K_{A}$ works as a coding for the unit ball

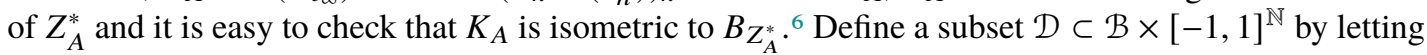

$$
\left(X, Y, \hat{A}, w^{*}\right) \in \mathcal{D} \Leftrightarrow w^{*} \in K_{A} .
$$

Because $Y \in \mathbb{B} \mapsto\left(e_{n}^{Y}\right)_{n} \in C(\Delta)^{\mathbb{N}}$ and $(A, z) \in \mathcal{B} \times C(\Delta) \mapsto\|z\|_{A}$ are Borel, it follows that $A \in \mathcal{B} \mapsto\left\|\alpha_{k} \times\left(z_{n}^{A}\right)\right\|_{A} \in \mathbb{R}$ is also Borel. Hence, $\mathcal{D}$ is Borel. ${ }^{7}$

Lemma 5.2. Let $\mathcal{B} \subset \mathcal{W}_{\mathrm{SB}, \mathrm{SD}}$ be Borel. The map

$$
A=(X, Y, \hat{A}) \in \mathcal{B} \mapsto K_{A} \in \mathcal{K}\left(B_{\ell_{\infty}}\right)
$$


is Borel. Moreover, for all $A \in \mathcal{B}$ there exists an isometry $i_{A}: K_{A} \rightarrow B_{Z_{A}^{*}}$ such that if $f=i_{A}\left(w^{*}\right)$, then $w_{k}^{*}=f\left(\alpha_{k} \times\left(z_{n}^{A}\right)\right) /\left\|\alpha_{k} \times\left(z_{n}^{A}\right)\right\|_{A}$ for all $k \in \mathbb{N}$.

Proof. The first statement is a simple consequence of [24, Theorem 28.8] and the second statement is a trivial consequence of the interpretation of $K_{A}$ as a coding for $B_{Z_{A}^{*}}$.

Because $K_{A}$ is a coding for the unit ball of $Z_{A}^{*}$, we must have a Borel way to realise the duality between $Z_{A}$ and $K_{A}$. The next two lemmas take care of this task.

Lemma 5.3. Let $\mathcal{B} \subset \mathcal{W}_{\mathrm{SB}, \mathrm{SD}}$ be Borel and let $i_{A}$ be as in Lemma 5.2. Let

$$
\mathcal{A}=\left\{\left(X, Y, \hat{A}, k, w^{*}\right) \in \mathcal{B} \times \mathbb{N} \times[-1,1]^{\mathbb{N}}: w^{*} \in K_{A}\right\}
$$

and define a map $\alpha: \mathcal{A} \rightarrow \mathbb{R}$ by

$$
\alpha\left(X, Y, \hat{A}, k, w^{*}\right)=\left\langle i_{A}\left(w^{*}\right), \alpha_{k} \times\left(z_{n}^{A}\right)\right\rangle
$$

for all $\left(X, Y, \hat{A}, k, w^{*}\right) \in \mathcal{A}$. Then $\mathcal{A}$ is a Borel set and $\alpha$ is a Borel map.

Proof. Because the assignment $A \in \mathcal{B} \rightarrow K_{A} \in \mathcal{K}\left([-1,1]^{\mathbb{N}}\right)$ is Borel by Lemma 5.2, it follows that $\mathcal{A}$ is Borel. In addition, because for all $\left(X, Y, \hat{A}, n, w^{*}\right) \in \mathcal{A}$ we have that

$$
\alpha\left(X, Y, \hat{A}, k, w^{*}\right)=w_{n}^{*}\left\|\alpha_{k} \times\left(z_{n}^{A}\right)\right\|_{A},
$$

the map $\alpha$ is clearly Borel.

Given $A=(X, Y, \hat{A}) \in \mathcal{B}, Z_{A}$ is defined as the interpolation space of the pair $\left(C(\Delta), W_{A}\right)$, and we can consider the standard inclusion $J_{A}: Z_{A} \rightarrow C(\Delta)$. Moreover, by 2' above, we have that $A\left(B_{X}\right) \subset W_{A} \subset B_{Z_{A}}$. Therefore, the map $j_{A}: X \rightarrow Z_{A}$ given by $j_{A}(x)=J_{A}^{-1}(A(x))$ is well defined and has norm at most 1 .

Lemma 5.4. Let $\mathcal{B} \subset \mathcal{W}_{\mathrm{SB}, \mathrm{SD}}$ be Borel, $i_{A}$ be as in Lemma 5.2 and $j_{A}$ be as above. Let

$$
\mathcal{A}^{\prime}=\left\{\left(X, Y, \hat{A}, x, w^{*}\right) \in \mathcal{B} \times C(\Delta) \times[-1,1]^{\mathbb{N}}: x \in X, w^{*} \in[-1,1]^{\mathbb{N}}\right\}
$$

and define a map $\alpha^{\prime}: \mathcal{A}^{\prime} \rightarrow \mathbb{R}$ by

$$
\alpha^{\prime}\left(X, Y, \hat{A}, x, w^{*}\right)=\left\langle i_{A}\left(w^{*}\right), j_{A}(x)\right\rangle
$$

for all $\left(X, Y, \hat{A}, x, w^{*}\right) \in \mathcal{A}^{\prime}$. Then $\mathcal{A}^{\prime}$ is a Borel set and $\alpha^{\prime}$ is a Borel map.

Proof. Notice that for each $\left(X, Y, \hat{A}, x, w^{*}\right) \in \mathbb{A}^{\prime}$,

$$
\alpha^{\prime}\left(X, Y, \hat{A}, x, w^{*}\right)=\lim _{j} w_{n_{j}}^{*}\left\|\alpha_{n_{j}} \times\left(z_{n}^{A}\right)\right\|_{A},
$$

where $\left(n_{j}\right)_{j}$ is any sequence in $\mathbb{N}$ so that $\alpha_{n_{j}} \times\left(z_{n}^{A}\right) \rightarrow j_{A}(x)$. Therefore,

$$
\begin{gathered}
\left\{\left(X, Y, \hat{A}, x, w^{*}\right) \in \mathcal{A}^{\prime}: \alpha^{\prime}\left(X, Y, \hat{A}, x, w^{*}\right) \in(a, b)\right\} \\
=\bigcup_{\delta \in \mathbb{Q}>0} \bigcap_{\varepsilon \in \mathbb{Q}_{>0}} \bigcup_{k \in \mathbb{N}}\left\{\left(X, Y, \hat{A}, x, w^{*}\right) \in \mathcal{A}^{\prime}:\left\|j_{A}(x)-\alpha_{k} \times\left(z_{j}^{A}\right)\right\|_{A}<\varepsilon,\right. \\
\left.\alpha\left(X, Y, \hat{A}, k, w^{*}\right) \in(a+\delta, b-\delta)\right\}
\end{gathered}
$$

where $\alpha$ is given by Lemma 5.3. This shows that $\alpha^{\prime}$ is Borel.

We now prove the main result of this subsection. 
Theorem 5.5. Let $\mathcal{B} \subset \mathcal{W}_{\mathrm{SD}}$ be a Borel set and let

$$
\mathcal{E}=\{(X, Y, \hat{A}, x) \in \mathcal{B} \times C(\Delta): x \in X\} .
$$

There are Borel maps

$$
\sigma: \mathcal{B} \rightarrow C(\Delta)^{\mathbb{N}} \text { and } \varphi: \mathcal{E} \rightarrow C(\Delta)
$$

such that, by setting $\varphi_{A}=(X, Y, \hat{A}, \cdot)$, we have that, for each $A=(X, Y, \hat{A}) \in \mathcal{B}$,

1. $\sigma(A)$ is a shrinking boundedly complete basic sequence,

2. $\operatorname{Im}\left(\varphi_{A}\right) \subset \overline{\operatorname{span}}\{\sigma(A)\}$ and $\varphi_{A}: X \rightarrow \overline{\operatorname{span}}\{\sigma(A)\}$ is a linear operator with norm at most 1 and

3. there exists a bounded operator $L: \operatorname{Im}\left(\varphi_{A}\right) \rightarrow Y$ so that $A=L \circ \varphi_{A}$. Moreover, $\|L\| \leqslant\left\|J_{A}\right\|$.

Proof. We follow the proof of [11, Theorem 4.6] closely. Let $H: \mathcal{K}\left([-1,1]^{\mathbb{N}}\right) \rightarrow C\left(\Delta,[-1,1]^{\mathbb{N}}\right)$ be the map given in Lemma 2.4 and $\alpha$ and $\alpha^{\prime}$ be the maps in Lemma 5.3 and Lemma 5.4, respectively. Fix a sequence $\left(n_{k}\right)_{k}$ in $\mathbb{N}^{\mathbb{N}}$ so that $\alpha_{n_{k}} \times\left(e_{j}^{Y}\right)=e_{k}^{Y}$ for all $k \in \mathbb{N}$. Notice that this sequence does not depend on $Y$. For each $A=(X, Y, \hat{A}) \in \mathcal{B}$, we define

$$
\sigma(A)=\left(\alpha\left(X, Y, \hat{A}, n_{k}, H\left(K_{A}\right)(\cdot)\right)\right)_{k} \cdot
$$

Because $A \in \mathcal{B} \mapsto K_{A} \in \mathcal{K}\left([-1,1]^{\mathbb{N}}\right)$ is Borel (Lemma 5.2), $\sigma$ is clearly Borel.

Claim 5.6. The sequence $\sigma(A)$ is 1-equivalent to $\left(z_{n_{k}}^{A}\right)_{k}$ for all $A \in \mathcal{B}$. In particular, $\sigma(A)$ is a shrinking boundedly complete basic sequence.

Proof. One only needs to notice that the assignment

$$
\alpha_{k} \times\left(z_{n_{k}}^{A}\right) \in Z_{A} \mapsto \alpha\left(X, Y, \hat{A}, k, H\left(K_{A}\right)(\cdot)\right) \in \overline{\operatorname{span}}\{\sigma(A)\}
$$

defines a surjective linear isometry $Z_{A} \rightarrow \overline{\operatorname{span}}\{\sigma(A)\}$. Because this follows exactly as in the proof of Theorem 4.6, we leave the details to the reader.

Define $\varphi: \mathcal{E} \rightarrow C(\Delta)$ by letting

$$
\varphi(X, Y, \hat{A}, x)=\alpha^{\prime}\left(X, Y, \hat{A}, x, H\left(K_{A}\right)(\cdot)\right),
$$

for all $(X, Y, \hat{A}, x) \in \mathcal{E}$. By Lemma 5.4, $\varphi$ is Borel. Given $A=(X, Y, \hat{A}) \in \mathcal{B}$, the $\operatorname{map} \varphi_{A}=\varphi(X, Y, \hat{A}, \cdot)$ is the composition of the map $j_{A}: X \rightarrow Z_{A}$ in Lemma 5.4 with the isometry $I_{A}: Z_{A} \rightarrow \overline{\operatorname{span}}\{\sigma(A)\}$, so $\operatorname{Im}\left(\varphi_{A}\right) \subset \overline{\operatorname{span}}\{\sigma(A)\}$. Moreover, because $\left\|j_{A}\right\| \leqslant 1$, the linear operator $\varphi_{A}$ has norm at most 1 . Finally, because $\varphi_{A}=I_{A} \circ j_{A}$, we have that $A=\left(J_{A} \circ I_{A}^{-1}\right) \circ \varphi_{A}$, so we are done.

Corollary 5.7. Let $\mathcal{B} \subset \mathcal{W}_{\mathrm{SB}, \mathrm{SD}}$ be Borel. There exists a Borel map $\Psi: \mathcal{B} \rightarrow \mathrm{SB}$ so that for all $A \in \mathcal{B}$,

1. $\Psi(A)$ is a reflexive space and

2. A factors through $\Psi(A)$.

Proof. Let $\mathcal{E}$ and $\varphi: \mathcal{B} \rightarrow C(\Delta)^{\mathbb{N}}$ be given by Theorem 5.5 and define $\Psi: \mathcal{B} \rightarrow$ SB by letting $\Psi(A)=\overline{\left\{\varphi\left(X, Y, \hat{A}, d_{n}(X)\right): n \in \mathbb{N}\right\}}$ for all $A=(X, Y, \hat{A}) \in \mathcal{B}$. Because $\varphi$ is Borel, so is $\Psi$, and the other properties follow from Theorem 5.5.

\subsection{Coding by Rational Spaces and Amalgamation}

We have seen in the previous subsection how to construct a Borel assignment $\sigma: \mathcal{B} \rightarrow C(\Delta)^{\mathbb{N}}$ so that every $A \in \mathcal{B}$ factors through a subspace of $\sigma(A)$. In this subsection, we code each $\sigma(A)$ as a subspace 
of a rational Banach space and use the amalgamation method presented in [25] in order to construct a single reflexive space containing every $\sigma(A)$.

Definition 5.8. Let $d \in \mathbb{N}$. A norm $\|\cdot\|$ on $\mathbb{R}^{d}$ is called rational if its unit ball is the convex hull of finitely many points whose coordinates in the standard basis of $\mathbb{R}^{d}$ are all rational.

Given $\bar{n}=\left(n_{j}\right)_{j} \in \mathbb{N}^{<\mathbb{N}}$, let us define a norm $\|\cdot\|_{\bar{n}}$ on $\mathbb{R}^{|\bar{n}|}$, where $|\bar{n}|$ denotes the length of the tuple $\bar{n}$. For that, for each $d \in \mathbb{N}$, fix an enumeration $\left(\|\cdot\|_{d, j}\right)_{j}$ of all monotone rational norms on $\mathbb{R}^{d}$. Then, given $\bar{n}=\left(n_{j}\right)_{j} \in \mathbb{N}^{<\mathbb{N}}$, define a norm $\|\cdot\|_{\bar{n}}$ on $\mathbb{R}^{|\bar{n}|}$ by letting the unit ball of $\left(\mathbb{R}^{|\bar{n}|},\|\cdot\|_{\bar{n}}\right)$ be

$$
B_{\left(\mathbb{R}^{\bar{n}},\|\cdot\|_{\bar{n}}\right)}=\overline{\operatorname{conv}}\left(\bigcup_{j=1}^{|\bar{n}|} B_{\left(\mathbb{R}^{j},\|\cdot\|_{j, n_{j}}\right)}\right) .
$$

If $\bar{n}=\left(n_{j}\right)_{j} \in \mathbb{N}^{\mathbb{N}}$, we define a norm $\|\cdot\|_{\bar{n}}$ on a subspace of $\mathbb{R}^{\mathbb{N}}$ analogously. To simplify notation, we denote the Banach spaces just defined by $\left(F_{\bar{n}},\|\cdot\|_{\bar{n}}\right)$ regardless of whether $\bar{n}$ is a finite tuple or not. For each such $\bar{n}$, we denote the standard basis of $\mathbb{R}^{\bar{n}}$ by $\left(r_{n}\right)_{n}$.

Let

$$
\text { mbs }=\left\{\bar{f} \in C(\Delta)^{\mathbb{N}}: \bar{f} \text { is a normalised monotone basic sequence }\right\},
$$

so mbs is a Borel subset of $C(\Delta)^{\mathbb{N}}$. We will now define a map $\psi:$ mbs $\rightarrow \mathbb{N}^{\mathbb{N}}$, which was implicitly defined in $\left[25\right.$, Section 6]. ${ }^{8}$

Fix a bijection $\pi=\left(\pi_{1}, \pi_{2}\right): \mathbb{N} \rightarrow \mathbb{N} \times \mathbb{N}$ so that both $\pi_{1}(m) \leqslant \pi_{1}(k)$ and $\pi_{2}(m) \leqslant \pi_{2}(k)$ imply $m \leqslant k$ (e.g., let $\pi$ be the bijection in [25, Definition 6.3]). For each $\bar{f}=\left(f_{n}\right)_{n} \in \operatorname{mbs}$, let $\left(f_{j, n}\right)_{j, n}$ denote the basis of $\ell_{2}(\overline{\operatorname{span}}\{\bar{f}\})$ so that $f_{j, n}$ belongs to the $j$ th copy of $\overline{\operatorname{span}}\{\bar{f}\}$ in $\ell_{2}(\overline{\operatorname{span}}\{\bar{f}\})$ and equals $f_{n}$. For each $i \in \mathbb{N}$, let $g_{i}=f_{\pi(i)}$, so $\left(g_{i}\right)_{i}$ is a monotone basis for $\ell_{2}(\overline{\operatorname{span}}\{\bar{f}\})$, for all $\bar{f} \in \operatorname{mbs}$.

We define the map $\psi: \operatorname{mbs} \rightarrow \mathbb{N}^{\mathbb{N}}$ as follows. Given $\bar{f} \in \operatorname{mbs}$ and $d \in \mathbb{N}$, let $n_{d} \in \mathbb{N}$ be the least natural number so that

$$
\begin{aligned}
\left(1-\frac{1}{2^{2 d+1}}\right)\left\|\sum_{i=1}^{d} a_{i} g_{i}\right\|_{\ell_{2}(\overline{\operatorname{span}}\{\bar{f}\})} & \leqslant\left\|\sum_{i=1}^{d} a_{i} r_{i}\right\|_{d, n_{d}} \\
& \leqslant\left(1-\frac{1}{2^{2 d+2}}\right)\left\|\sum_{i=1}^{d} a_{i} g_{i}\right\|_{\ell_{2}(\overline{\operatorname{span}}\{\bar{f}\})}
\end{aligned}
$$

for all $\left(a_{i}\right)_{i} \in \mathbb{R}^{d}$. Such $n_{d}$ exists because $\left(g_{i}\right)_{i}$ is a monotone basis of $\ell_{2}(\overline{\operatorname{span}}\{\bar{f}\})$. Set $\psi(\bar{f})=\left(n_{d}\right)_{d}$; this map is clearly Borel.

It follows from [25, Lemma 6.5] that the assignment $g_{i} \mapsto r_{i}$ defines an isomorphism between $\ell_{2}(\overline{\operatorname{span}}\{\bar{f}\})$ and $F_{\psi(\bar{f})}$ for all $\bar{f} \in$ mbs. For each $j \in \mathbb{N}$ and $\bar{f} \in$ mbs, we denote the canonical inclusion of $\overline{\operatorname{span}}\{\bar{f}\}$ onto its $j$ th copy in $F_{\psi(\bar{f})}$ by $U_{\bar{f}, m}$. Precisely, $U_{\bar{f}, m}: \overline{\operatorname{span}}\{\bar{f}\} \rightarrow F_{\psi(\bar{f})}$ is the linear map given by

$$
U_{\bar{f}, j}\left(f_{n}\right)=r_{\pi^{-1}(j, n)}
$$

for all $n \in \mathbb{N}$.

Lemma 5.9. Let $\psi: \mathrm{mbs} \rightarrow \mathbb{N}^{\mathbb{N}}$ be the Borel map defined above. Then, for all $\bar{f} \in \mathrm{mbs}$,

1. $F_{\psi(\bar{f})}$ is isomorphic to $\ell_{2}(\overline{\operatorname{span}}\{\bar{f}\})$,

2. if $\bar{f}$ is shrinking, then $\left(r_{n}\right)_{n}$ is a monotone shrinking basis for $F_{\psi(\bar{f})}$ and 
3. the map $U_{\bar{f}}: \overline{\operatorname{span}}\{\bar{f}\} \rightarrow F_{\psi(\bar{f})}$ given by

$$
U_{\bar{f}}(x)=\frac{\sqrt{3}}{2} \cdot \sum_{j \in \mathbb{N}} \frac{1}{2^{j-1}} U_{\bar{f}, j}(x)
$$

is an isometry and its image is a 1-complemented subspace of $F_{\psi(\bar{n})}$.

Proof. The map $\psi$ is clearly Borel, and the other statements are precisely the content of [25, Lemmas 6.5 and 6.7].

Proof of Theorem 5.1. Let $\mathcal{B} \subset \mathcal{W}_{\mathrm{SB}, \mathrm{SD}}$ be a Borel subset, and let $\mathcal{E} \subset \mathcal{B} \times C(\Delta), \sigma: \mathcal{B} \rightarrow C(\Delta)^{\mathbb{N}}$ and $\varphi: \mathcal{E} \rightarrow C(\Delta)$ be as in Theorem 5.5. Without loss of generality, we can assume that $\sigma(A)$ is normalised, for each $A \in \mathcal{B}$. Let $\psi:$ mbs $\rightarrow \mathbb{N}^{\mathbb{N}}$ be given by Lemma 5.9. Then $\mathbb{A}=\psi(\sigma(\mathcal{B}))$ is an analytic subset of the Baire space $\mathbb{N}^{\mathbb{N}}$. Hence, there exists a pruned tree $T$ on $\mathbb{N} \times \mathbb{N}$ so that $\mathbb{A}=p([T])$, where $p: \mathbb{N}^{\mathbb{N}} \times \mathbb{N}^{\mathbb{N}} \rightarrow \mathbb{N}^{\mathbb{N}}$ denotes the projection onto the first coordinate (see [24, Theorem 25.2]).

Define a norm $\|\cdot\|$ on $c_{00}(T)$ by letting

$$
\|x\|=\sup _{\beta \in[T]}\left\|\sum_{t<\beta} x(t) r_{|t|}\right\|_{p(\beta)}
$$

for each $x=(x(t))_{t \in T} \in c_{00}(T)$. Let $E$ be the completion of $c_{00}(T)$ under the norm $\|\cdot\|$, and let $\left(e_{t}\right)_{t \in T}$ denote the canonical basis of $c_{00}(T)$; that is, $e_{t}(t)=1$ and $e_{t}(s)=0$ for all $s \neq t$. Therefore, $\left(e_{t}\right)_{t \in T}$ is a basis for $E$ (see [25, Definition 3.1] for details).

Clearly, if $\bar{n}=\psi(\sigma(A))$ for some $A \in \mathcal{B}$, then $F_{\bar{n}}$ is isometric to a subspace of $E$. Indeed, let $\bar{m} \in \mathbb{N}^{\mathbb{N}}$ be so that $\beta=(\bar{n}, \bar{m}) \in[T]$. Then the assignment $r_{i} \mapsto e_{\beta\lceil i}$ defines an isometry between $F_{\bar{n}}$ and

$$
E_{\beta}=\left\{x=(x(t))_{t \in T} \in E: t \nless \beta \Rightarrow x(t)=0\right\} .
$$

However, we need a $\sigma\left(\Sigma_{1}^{1}\right)$-measurable way of choosing such $\bar{m} \in \mathbb{N}$. For that, notice that $[T]$ is a closed subset of $\mathbb{N}^{\mathbb{N}} \times \mathbb{N}^{\mathbb{N}}$, so the Jankov-von Neumann uniformisation theorem gives a $\sigma\left(\Sigma_{1}^{1}\right)$-measurable map $\theta: \mathbb{A} \rightarrow[T]$ that uniformises $[T]$ (see [24, Theorem 18.1]); that is, $p(\theta(\beta))=\beta$ for all $\beta \in \mathbb{A}$. For each $A \in \mathcal{B}$, define $t_{A}=\theta(\psi(\sigma(A)))$. Therefore, $F_{\psi(\sigma(A))}$ is canonically isometric to $E_{t_{A}}$ for all $A \in \mathcal{B}$.

Define $W=\overline{\operatorname{conv}} \bigcup_{\beta \in[T]} B_{E_{\beta}}$, and let $Z=\Delta_{2}(E, W)$. Because $Z$ is separable, by fixing an isometric copy of $Z$ in SB, we can assume without loss of generality that $Z \in \mathrm{SB}$.

Claim 5.10. The interpolation space $Z$ is reflexive.

Proof. By Theorem 5.5, it follows that $\sigma(A)$ is a boundedly complete shrinking basis for all $A \in \mathcal{B}$, so $\overline{\operatorname{span}}\{\sigma(A)\}$ is reflexive. Hence, by Lemma 5.9, $F_{\psi(\sigma(A))}$ is reflexive for all $A \in \mathcal{B}$. Then $E_{\beta}$ is reflexive for all $\beta \in[T]$ and [25, Proposition 4.6] gives us that $Z$ is reflexive.

Because $B_{E_{\beta}} \subset W$ for all $\beta \in[T]$, the natural inclusion $i_{\beta}: E_{\beta} \rightarrow Z$ is well defined and bounded for all $\beta \in[T]$. Moreover, by [25, Lemma 4.2 and Fact 4.4], there exists $c>0$ so that the map $x \in E_{\beta} \mapsto \operatorname{ci}_{\beta}(x) \in Z$ is an isometry for all $\beta \in[T]$. For each $A \in \mathcal{B}$ and $k \in \mathbb{N}$, let $t_{A, k} \in T$ be the initial segment of $t_{A}$ with length $k$ and define a map $I_{A}: F_{\psi(\sigma(A))} \rightarrow Z$ by

$$
I_{A}: \sum_{k \in \mathbb{N}} a_{k} r_{k} \in F_{\psi(\sigma(A))} \mapsto c i_{t_{A}}\left(\sum_{k \in \mathbb{N}} a_{k} e_{t_{A, k}}\right) \in Z
$$

Then $I_{A}$ is an isometric embedding for all $A \in \mathcal{B}$. 
Let $\mathcal{D}$ be as in the statement of Theorem 5.1. We define the desired maps $\Phi: \mathcal{D} \rightarrow Z$ and $\Psi: \mathcal{B} \rightarrow \mathrm{SB}(Z)$ by letting

$$
\Phi(X, Y, \hat{A}, x)=I_{A}\left(U_{\sigma(A)}(\varphi(X, Y, \hat{A}, x))\right)
$$

for all $(X, Y, \hat{A}, x) \in \mathcal{D}$ and

$$
\Psi(X, Y, \hat{A})=\overline{\operatorname{span}}\left\{\Phi\left(X, Y, \hat{A}, d_{n}(X)\right): n \in \mathbb{N}\right\}
$$

for all $(X, Y, \hat{A}) \in \mathcal{B}$, where $\left(d_{n}\right)_{n}$ are the Kuratowski and Ryll-Nardzewski Borel selectors (see Subsection 2.2).

Because $\varphi_{A}=\varphi(X, Y, \hat{A}, \cdot): X \rightarrow \overline{\operatorname{span}}\{\sigma(A)\}$ is a bounded linear map with norm at most 1 and both $I_{A}$ and $U_{\sigma(A)}$ are isometries, it follows that $\Phi_{A}=\Phi(X, Y, \hat{A}, \cdot): X \rightarrow Z$ is a bounded linear map with norm at most 1 for all $A \in \mathcal{B}$. Therefore, (2) holds. Moreover, this gives that $\Psi(X, Y, \hat{A}) \in \operatorname{SB}(Z)$, so $\Psi$ is well defined.

Given $A=(X, Y, \hat{A}) \in \mathcal{B}$, Theorem 5.5 gives a bounded operator $L: \operatorname{Im}\left(\varphi_{A}\right) \rightarrow Y$ so that $A=L \circ \varphi_{A}$ and $\|L\| \leqslant\left\|J_{A}\right\|$. Hence, because $\Phi_{A}=I_{A} \circ U_{\sigma(A)} \circ \varphi_{A}$, it follows that

$$
A=\left(L \circ U_{\sigma(A)}^{-1} \circ I_{A}^{-1}\right) \circ \Phi_{A} .
$$

Therefore, $A$ factors through $\Psi(X, Y, \hat{A})$ for all $(X, Y, \hat{A}) \in \mathcal{B}$ and (3) holds.

Claim 5.11. The maps $\Phi$ and $\Psi$ are $\sigma\left(\Sigma_{1}^{1}\right)$-measurable.

Proof. By the definition of $\Psi$, it is enough to show that $\Phi$ is $\sigma\left(\Sigma_{1}^{1}\right)$-measurable. Firstly, notice that, because $A \in \mathcal{B} \rightarrow t_{A} \in \mathbb{N}^{\mathbb{N}} \times \mathbb{N}^{\mathbb{N}}$ is $\sigma\left(\Sigma_{1}^{1}\right)$-measurable, the assignment

$$
A \in \mathcal{B} \mapsto\left(i_{t_{A}}\left(e_{t_{A, k}}\right)\right)_{k} \in C(\Delta)^{\mathbb{N}}
$$

is also $\sigma\left(\Sigma_{1}^{1}\right)$-measurable.

Let $U \subset C(\Delta)$ be an open ball. To simplify notation, for each $A=(X, Y, \hat{A}) \in \mathcal{B}$, let $\sigma(A)=\left(\sigma_{A, i}\right)_{i}$. By the definition of $\Phi_{A}$, for every $\varphi_{A}(x)=\sum_{i=1}^{k} a_{i} \sigma_{A, i} \in \overline{\operatorname{span}}\{\sigma(A)\}$, we have

$$
\Phi_{A}(x)=c \cdot \frac{\sqrt{3}}{2} \sum_{j \in \mathbb{N}} \frac{1}{2^{j-1}} \sum_{i=1}^{k} a_{i} i_{t_{A}}\left(e_{t_{A, \pi^{-1}(j, i)}}\right) .
$$

Then

$$
\begin{aligned}
\Phi_{A}(x) \in U \Leftrightarrow & \exists \delta \in \mathbb{Q}>0, \exists k \in \mathbb{N}, \exists a_{1}, \ldots a_{k} \in \mathbb{Q} \\
& \left(\left\|\varphi_{A}(x)-\sum_{i=1}^{k} a_{i} \sigma_{A, i}\right\|<\delta\right) \wedge(\forall m \in \mathbb{N} \\
& \left.\left\|d_{m}(C(\Delta))-c \cdot \frac{\sqrt{3}}{2} \sum_{j \in \mathbb{N}} \frac{1}{2^{j-1}} \sum_{i=1}^{k} a_{i} i_{t_{A}}\left(e_{t_{A, \pi^{-1}(j, i)}}\right)\right\|<\delta \rightarrow d_{m}(C(\Delta)) \in U\right) .
\end{aligned}
$$

This shows that $\Phi$ is $\sigma\left(\Sigma_{1}^{1}\right)$-measurable. 
Because we clearly have that $\Phi_{A}(X)=\Psi(X)$ for all $(X, Y, \hat{A}) \in \mathcal{B},(1)$ holds and the proof is finished.

Remark 5.12. Notice that it is immediate from the proof of Theorem 5.1 that the map $L$ in Theorem 5.1(3) satisfies $\|L\| \leq\left\|J_{A}\right\|$. Precisely, see the paragraph preceding Claim 5.11.

P. Dodos and V. Ferenczi showed that REFL $=\{X \in \mathrm{SB}: X$ is reflexive $\}$ is a strongly bounded class of Banach spaces. Theorem 5.1 allow us to strengthen this result. Precisely, we have the following corollary.

Corollary 5.13. Say $\mathbb{B} \subset$ REFL is Borel. There exists $a Z \in R E F L$ with a basis and a $\sigma\left(\Sigma_{1}^{1}\right)$-measurable map $\Psi: \mathbb{B} \rightarrow \mathrm{SB}(Z)$ such that $X \equiv \Psi(X)$, for all $X \in \mathbb{B}$. Moreover, setting

$$
\mathbb{E}=\{(X, x) \in \mathbb{B} \times C(\Delta) \mid x \in X\},
$$

there exists a $\sigma\left(\Sigma_{1}^{1}\right)$-measurable map

$$
\psi: \mathbb{E} \rightarrow Z
$$

such that, letting $\psi_{X}=\psi(X, \cdot)$, we have that $\psi_{X}: X \rightarrow Z$ is an isometric embedding for all $X \in \mathbb{B}$.

Proof. Let $\mathcal{B}=\left\{\left(X, X,\left(d_{n}(X)\right)_{n}\right) \in \mathcal{L}: X \in \mathbb{B}\right\}$. Notice that $\left(d_{n}(X)\right)_{n}$ codes the identity operator on $X$. Because $\mathbb{B} \subset$ REFL, we have that $\mathcal{B} \subset \mathcal{W}_{\mathrm{SD}}$. Let $Z \in \mathrm{REFL}, \Psi: \mathcal{B} \rightarrow \operatorname{SB}(Z), \mathcal{D}$, and $\Phi: \mathcal{D} \rightarrow Z$ be given by Theorem 5.1. Moreover, Theorem 5.1 also gives that, for all $X \in \mathbb{B}$, there exists a bounded operator $L: \Psi(X) \rightarrow Y$ so that $\operatorname{Id}_{X}=L \circ \Phi_{\mathrm{Id}_{X}}$ and $\|L\| \leqslant\left\|J_{\mathrm{Id}_{X}}\right\|$ (see Remark 5.12). Because $\left\|J_{\mathrm{Id}_{X}}\right\| \leqslant 1$ for all $X \in \mathbb{B}$, we have $\|L\| \leqslant 1$. Because $\left\|\Phi_{\mathrm{Id}_{X}}\right\| \leqslant 1$, we conclude that $\Phi_{\mathrm{Id}_{X}}: X \rightarrow \Psi(X)$ is an isometric embedding.

\section{Concluding Remarks and Questions}

We finish this article with some natural questions that are left open. Firstly, it would be very interesting to get rid of $\sigma\left(\Sigma_{1}^{1}\right)$-measurability in the theorems in Subsection 5.2. Because an arbitrary analytic subset of $\mathbb{A} \subset \mathbb{N}^{\mathbb{N}} \times \mathbb{N}^{\mathbb{N}}$ may not have a Borel uniformisation even if $p(\mathbb{A})=\mathbb{N}^{\mathbb{N}}$, a proof for that will probably require some new ideas and a different amalgamation method.

\section{Question 6.1. Do the Borel-measurable versions of Theorem 5.1 and Corollary 5.13 hold?}

As noticed in the Introduction, if $\mathcal{C}$ is a collection of weakly compact operators containing the identity on a Banach space without the bounded approximation property, then there is no hope of finding a reflexive space $Z$ with a Schauder basis so that all members of $\mathcal{C}$ factor through $A$. Although Theorem 4.3 gives us some conditions on $\mathcal{C}$ for which a positive answer holds, we are very far from completely understanding what the precise conditions are for such $Z$ to exist.

Question 6.2. Let $\mathcal{C} \subset \mathcal{W}$ be a set of weakly compact operators so that each element of $\mathcal{C}$ factors through a reflexive Banach space with a Schauder basis. Is $\mathcal{C}$ strongly bounded?

Recall that if a Banach space $Y$ has a basis so that $Y^{*}$ is separable and has the bounded approximation property, then $Y$ has a shrinking basis [22]. Hence, [9, Theorem 25] applies to a collection $\mathcal{C}$ whose domain spaces satisfies this property. However, if $Y^{*}$ is not assumed to be separable, the question remains open. Precisely, the following was asked by W.B. Johnson in MathOverflow [21].

Question 6.3. Let $A: X \rightarrow Y$ be weakly compact and suppose that $Y$ has a basis and $Y^{*}$ has the bounded approximation property. Is there a reflexive $Z$ with a basis so that $A$ factors through $Z$ ?

Our final problem is related to an open problem of P. Dodos from [17]. For the definition of coanalytic ranks, see [16, Appendix A]. 
Question 6.4. Let $\phi$ be a coanalytic rank on $\mathcal{L}_{\mathrm{SD}}$ and let $\mathcal{A}$ be an analytic subset of dual operators in $\mathcal{L}$. Suppose that for each $A \in \mathcal{A}$ there is a countable ordinal $\xi_{A}$ so that

$$
\sup \left\{\phi(B): B^{*} \text { is isomorphic to } A\right\}<\xi_{A} \text {. }
$$

Is the set

$$
\mathcal{A}_{*}=\left\{B \in \mathcal{L}_{\mathrm{SD}}: \exists A \in \mathcal{A} \text { where } B^{*} \text { is isomorphic to } A\right\}
$$

analytic?

Acknowledgements. Part of this article was written while the third named author was visiting the Universidade de São Paulo (USP), Brazil, in May 2019. This author thanks Valentin Ferenczi and Christina Brech for their hospitality. The second author acknowledges the financial support of Washington \& Lee's Summer Lenfest Grant.

\section{References}

[1] J. Abramovič, 'Weakly compact sets in topological K-spaces', Teor. Funkciŭ Funkcional. Anal. i Priložen. 15 (1972), $27-35$.

[2] F. Albiac and N. J. Kalton, Topics in Banach Space Theory, Vol. 233 of Graduate Texts in Mathematics (Springer, New York, 2006).

[3] S. A. Argyros and P. Dodos, 'Genericity and amalgamation of classes of Banach spaces', Adv. Math. 209(2) (2007), 666-748.

[4] K. Beanland and R. M. Causey, 'On a generalization of Bourgain's tree index', Houston J. Math. 44(1) (2018), 201-208.

[5] K. Beanland and R. M. Causey, 'Quantitative factorization of weakly compact, Rosenthal, and $\xi$-Banach-Saks operators', Math. Scand. 123(2) (2018), 297-319.

[6] K. Beanland and R. M. Causey, 'Genericity and universality for operator ideals', Q. J. Math. 71(3) (2020), 1081-1129.

[7] K. Beanland, R. Causey, D. Freeman and B. Wallis, 'Classes of operators determined by ordinal indices', J. Funct. Anal. 271(6) (2016), 1691-1746.

[8] K. Beanland and D. Freeman, 'Ordinal ranks on weakly compact and Rosenthal operators', Extracta Math. 26(2) (2011), 173-194.

[9] K. Beanland and D. Freeman, 'Uniformly factoring weakly compact operators', J. Funct. Anal. 266(5) (2014), $2921-2943$.

[10] B. Bossard, 'A .32coding of separable Banach spaces. Analytic and coanalytic families of Banach spaces', Fund. Math. 172(2) (2002), 117-152.

[11] B. M. Braga, 'Duality on Banach spaces and a Borel parametrized version of Zippin's theorem', Ann. Inst. Fourier (Grenoble) 65(6) (2015), 2413-2435.

[12] R. M. Causey and K. V. Navoyan, ' $\zeta$-completely continuous operators and $\zeta$-Schur Banach spaces', J. Funct. Anal. 276(7) (2019), 2052-2102.

[13] M. Cúth, M. Doležal, M. Doucha and K. Ondřej, 'Polish spaces of Banach spaces. Complexity of isometry classes and generic properties', preprint arXiv:1912.03994.

[14] M. Cúth, M. Doucha and K. Ondřej, 'Complexity of distances: reductions of distances between metric and Banach spaces', preprint arXiv:2004.11752.

[15] W. J. Davis, T. Figiel, W. B. Johnson and A. Pełczyński, 'Factoring weakly compact operators', J. Funct. Anal. 17 (1974), 311-327.

[16] P. Dodos, Banach Spaces and Descriptive Set Theory: Selected Topics, Vol. 1993 of Lecture Notes in Mathematics (SpringerVerlag, Berlin, 2010).

[17] P. Dodos, 'Definability under duality', Houston J. Math. 36(3) (2010), 781-792.

[18] P. Dodos and V. Ferenczi, 'Some strongly bounded classes of Banach spaces', Fund. Math. 193(2) (2007), 171-179.

[19] T. Figiel, W. B. Johnson and L. Tzafriri, 'On Banach lattices and spaces having local unconditional structure, with applications to Lorentz function spaces', J. Approx. Theory 13 (1975), 395-412. Collection of articles dedicated to G. G. Lorentz on the occasion of his 65 th birthday.

[20] G. Godefroy and J. Saint-Raymond, 'Descriptive complexity of some isomorphism classes of Banach spaces', J. Funct. Anal. 275(4) (2018), 1008-1022.

[21] W. B. Johnson, URL: https://mathoverflow.net/questions/240472/davis-figiel-johnson-and-pelczynski-factorization-throughspaces-with-a-bases.

[22] W. B. Johnson, H. P. Rosenthal and M. Zippin, 'On bases, finite dimensional decompositions and weaker structures in Banach spaces', Israel J. Math. 9 (1971), 488-506.

[23] W. B. Johnson and A. Szankowski, 'Complementably universal Banach spaces. II', J. Funct. Anal. 257(11) (2009), 33953408.

[24] A. S. Kechris, Classical Descriptive Set Theory, Vol. 156 of Graduate Texts in Mathematics (Springer, New York, 1995).

[25] O. Kurka, 'Amalgamations of classes of Banach spaces with a monotone basis', Studia Math. 234(2) (2016), 121-148. 
[26] O. Kurka, 'Zippin's embedding theorem and amalgamations of classes of Banach spaces', Proc. Amer. Math. Soc. 144(10) (2016), 4273-4277.

[27] A. Pełczyński, 'Universal bases', Studia Math. 32 (1969), 247-268.

[28] W. Szlenk, 'The non-existence of a separable reflexive Banach space universal for all separable reflexive Banach spaces', Studia Math. 30 (1968), 53-61. 